\title{
LA MINERÍA AURÍFERA ROMANA EN EL NORDESTE DE LUSITANIA: LAS CAVENES DE EL CABACO (SALAMANCA)
}

POR

\author{
M. RUIZ DEL ÁRBOL \\ Universidad Complutense de Madrid \\ F.-J. SÁNCHEZ-PALENCIA \\ Centro de Estudios Históricos del CSIC ${ }^{1}$
}

\begin{abstract}
RESUMEN
El estudio de las minas de oro romanas de Las Cavenes proporciona nuevos datos para el conocimiento del desarrollo de la minería romana en este sector lusitano de Hispania tan poco estudiado hasta ahora. En el artículo consideramos el papel que pudo jugar esta minería en la nueva ordenación y ocupación del suelo que implicó la reorganización provincial de comienzos del imperio y, a una escala regional, en la reorganización que la documentación antigua apunta en torno a Salmantica, Bletisama y Mirobriga. Los trabajos desarrollados por nuestro equipo han proporcionado evidencias arqueológicas que permiten plantear tanto el estudio de las labores mineras como su incidencia en la puesta en marcha y revalorización de los recursos agropecuarios en la zona.
\end{abstract}

\section{SUMMARY}

The study of Roman gold mines of Las Cavenes gives new data on the developement of Roman mining in this Lusitanian part of Hispania so little studied until now. We consider the role that mining could have played in the new territorial planning and occupation, that provoked the provincial reorganisation of the beginning of the Empire; and at a regional level, the one documented in Salmantica, Bletisama and Mirobriga. The work carried out by our team has gathered archaeological evidence for the study of mining activities and for their incidence on the starting and the revaluation of farming resources in the region.

Con el nombre de Cavenes se conocen un conjunto de desmontes localizados al pie de la Sierra de Francia, en el municipio de El Cabaco, situado al sur de la provincia de Salamanca (figs.1 y 2). La existencia de estas labores mineras fue puesta ya de manifiesto por Gómez-Moreno, que las incluye en

1 Este trabajo ha sido realizado en el marco de los proyectos de investigación: Paisajes Antiguos en la Península Ibérica: teoría y práctica de la Arqueología del Paisaje (PB971129 de la DGICYT) y Zona Arqueológica de Las Cavenes (Ayuntamiento de El Cabaco - DRG, s.a. - CSIC, financiado por la Junta de Castilla y León). Estos proyectos se desarrollan en el Depto. de Historia Antigua y Arqueología del Centro de Estudios Históricos del CSIC. su Catálogo Monumental de España $(1967,53)$ siguiendo las noticias del ingeniero Toribio Cáceres, a partir de las cuales las describe relacionándolas con las minas de oro del Noroeste, en concreto con las del Bierzo. Los desmontes fueron reconocidos también por Morán (1946, 29-31) que enumera las distintas Cavenes y realiza una breve descripción de las mismas, a las que considera indicios de una explotación en época romana para extraer manganeso, «que sirvió desde la antigüedad para la industria del vidrio». Más tarde, Maluquer $(1956,53)$, menciona en esta misma zona la existencia de una serie de asentamientos de cronología romana que pone en relación con la explotación del mineral - esta vez de hierro- de las Cavenes ${ }^{2}$. Los últimos estudios realizados sobre minería en la Península Ibérica ${ }^{3}$ identifican claramente las labores de Las Cavenes como pertenecientes a la minería aurífera romana, algo ya admitido por todos los autores (como resumen: TIR, K-29, 1991 s.v. Cavenes, Las; Fuente de la Mora y Maíllo, El; MGE, h. n 527, Tamames, 1990, 84).

Nuestros trabajos se han articulado en torno al estudio de estas labores mineras, cuya zona principal se extiende a lo largo de un área de unos $14 \mathrm{~km}^{2}$ al oeste-suroeste del pueblo de El Cabaco (figs. 2 y 3 ). En concreto, la zona de labores más densa, en la que hemos centrado hasta ahora la mayor parte de las actuaciones, ocupa los bordes de una llanura amesetada a lo largo de una extensión de $4 \times 1$ ' $5 \mathrm{~km}$. Esta

\footnotetext{
${ }^{2}$ Los dos autores también señalan una serie de emplazamientos relacionados con las labores. Morán $\left(1940,14, \mathrm{n}^{\circ}\right.$ 35-40) identifica como castro prehistórico el Teso de las Tiendas, y como romanos La Mesita, La Corona, La Cabezuela, La Tarayuela y el Castro Mirón. En 1946 (pp. 29-31) vincula todos ellos a la minería romana pero no menciona La Cabezuela y añade el Llano Redondo; apunta el hallazgo de ladrillos típicamente romanos en Las Vagüeras y de una pesa en la Fuente de la Mora. Maluquer $(1956,53)$, aunque cita el trabajo de Morán, destaca sin embargo como lugar principal La Fuente de la Mora, donde sitúa todos los hallazgos romanos.

3 Sánchez-Palencia, 1983, 464 y 1989, 48 ss; SánchezPalencia y Pérez, 1989, 17, nº 6; Recursos Minerales, 1988, 49; Domergue, 1987, II, 457 y 1990, 41, 201.
} 


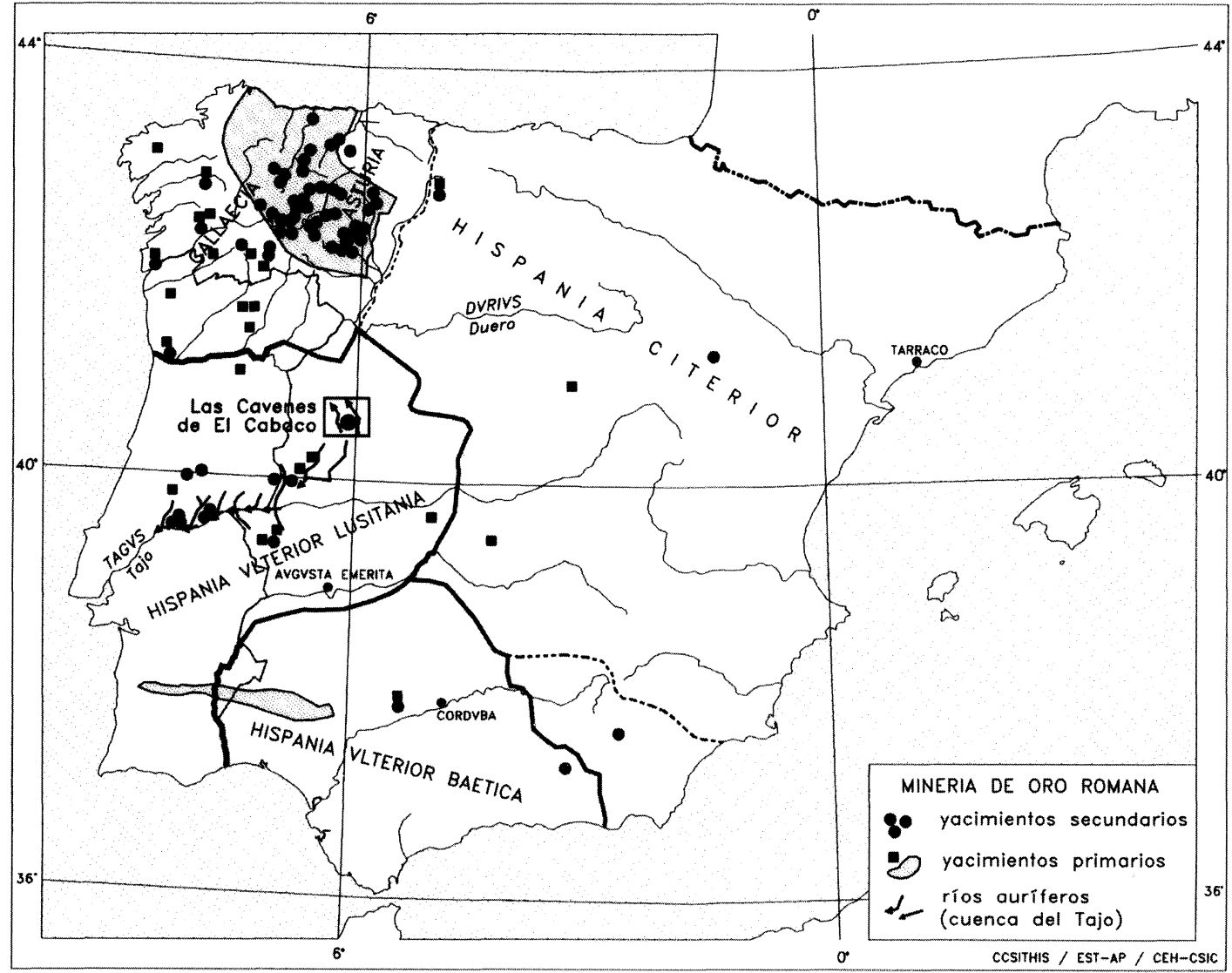

Fig. 1. - Situación de Las Cavenes de El Cabaco en el contexto de la minería de oro romana en la Península Ibérica.

meseta se halla delimitada por el arroyo del Zarzosi1 lo al oeste y por el río Gabín al este. Ambos son afluentes del arroyo del Zarzoso que, a su vez, es uno de los que dan origen al río Yeltes, afluente del Huebra y éste del Duero ${ }^{4}$. El desarrollo de la explotación minera ha hecho que los desmontes se dispongan de forma prácticamente lineal en los bordes de la meseta. La dedicación tradicional del suelo en esta región hasta la actualidad ha permitido que se conserven en el paisaje, de forma excepcional, las diversas estructuras del laboreo minero: la red hidráulica, los vaciados mineros y las zonas de evacuación y acumulación de los estériles resultantes del proceso de extracción del mineral. Estas condiciones han permitido llevar a cabo, a partir de la interpretación de la fotografía aérea, de la realización de una detallada topografía del terreno y de la excavación de algunas de las estructuras mineras, un estudio completo de las labores y del desarrollo de su explotación.

${ }^{4}$ Sus referencias geográficas son las siguientes: las coordenadas UTM que delimitan las labores son: 29TQE 910$980 \mathrm{~N}$; 410-430 O; coordenadas geográficas: $40^{\circ} 32$ ' $10^{\prime \prime}$ $40^{\circ} 35^{\prime} 50$ 'N; 06 08' 00"'- 06 09' 20" O; altitud 950-1070 m; MTN hojas: 527-I, Tamames y 527-III, El Maíllo.
1. La ReEStRUCtURACión PROVINCIAL DE COMIENZOS DEl Principado. El inicio de las explotaciones AURÍFERAS LUSITANAS

Las labores de El Cabaco forman parte de un conjunto más amplio de explotaciones que se extiende a lo largo de la cuenca sedimentaria de Ciudad Rodrigo, al noreste de Lusitania, en pleno territorio vetón (fig. 1) ${ }^{5}$. Domergue (1990, 40 ss) las incluye en la gran región minera aurífera del noroeste, agrupándolas junto a las explotaciones localizadas en las tres provincias portuguesas que forman la zona de Beiras - dentro de lo que denomina «distrito de Beiras»- La existencia de estas explotaciones mineras ha sido considerada en algunos trabajos y señalada como uno de los indicadores que evidencian la complejidad de la ocupación romana en la provincia (Santonja, 1991, 29), pero salvo estas breves menciones en estudios generales - o las referencias aisladas proporcionadas por Morán y Maluquer- no se

5 Otros indicios de esta minería en la zona se encuentran a lo largo del río Tenebrilla, en el municipio vecino de $\mathrm{El}$ Maíllo. 


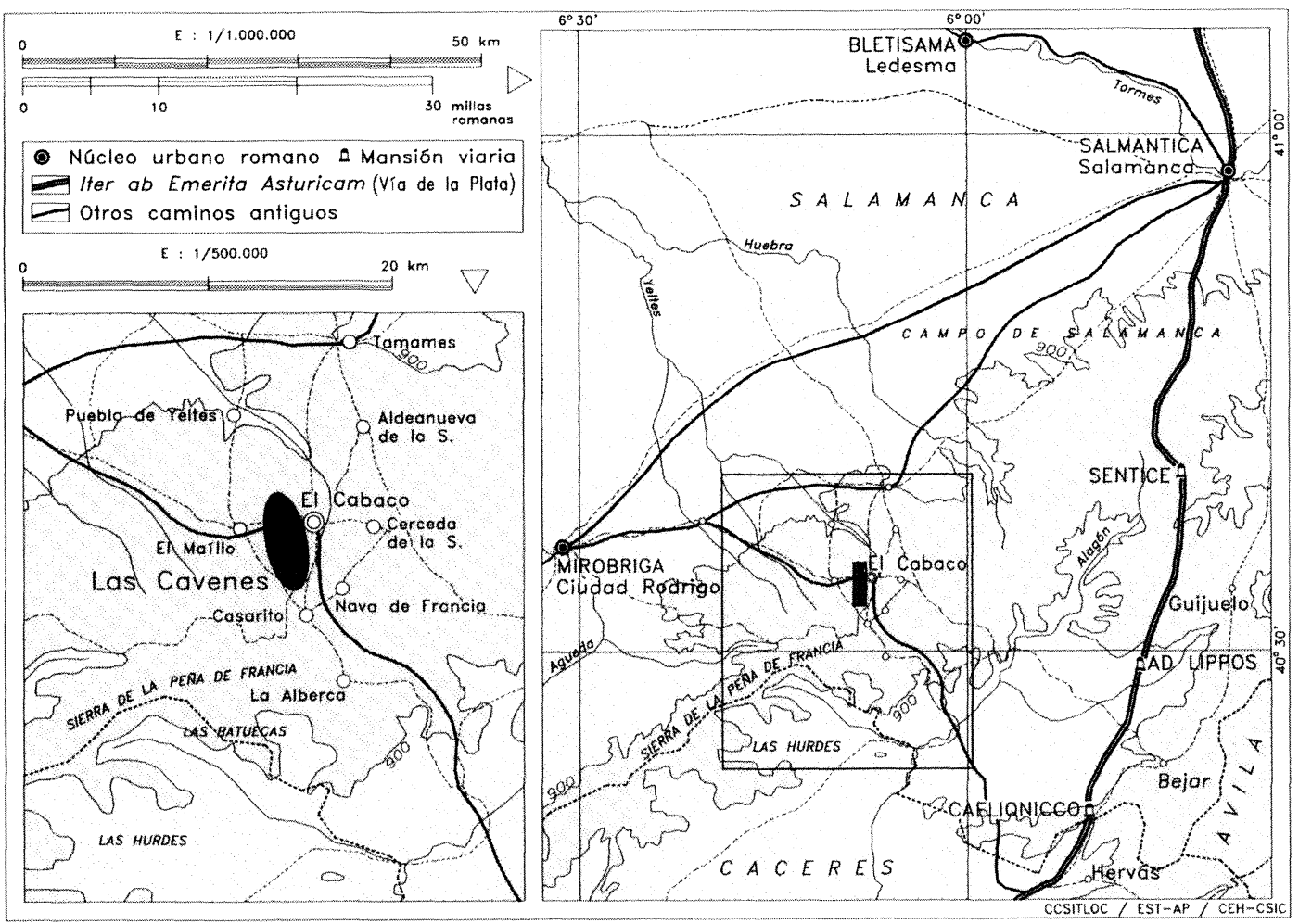

Fig. 2.-Situación de Las Cavenes de El Cabaco a escala comarcal.

ha prestado atención al desarrollo de esta minería y a su relación con la reorganización y estructuración de estos territorios en época romana. Algunos autores han vinculado la explotación de los yacimientos mineros del suroeste de la provincia con la distribución del poblamiento prerromano en esa zona, llegando a afirmar que «la minería debe haber contribuido también a la concentración de la población en la zona occidental de la provincia durante los siglos V-I a.C.» e incluyendo las minas de El Cabaco y El Mállo entre los factores que favorecen esta concentración, ya que «la existencia en un mismo área de varios castros próximos geográficamente (...) parece indicar el intento de explotar por parte de esta población los recursos mineros de esta zona» dentro de una estructura productiva calificada como «más atomizada» (Salinas, 1992-93, 179-80).

Gran parte de los autores que han tratado el tema admiten que la expedición de D. Junio Bruto, el Galaico, en el 136 a.C., supuso la incorporación definitiva a la Hispania romana del territorio situado al norte del Tajo, desplazándose así la frontera en la Meseta hasta la línea del Duero. Aunque esta zona no fue escenario de ninguna de las grandes operaciones de conquista, se vio afectada a lo largo de todo lo que quedaba de siglo y parte del siguien- te por diversos conflictos, ya fuesen de carácter local o regional, es decir, de enfrentamiento directo y más o menos coyuntural entre los pueblos indígenas y el poder romano, o inscritos en el marco de las luchas civiles de alcance global que caracterizaron en buena medida el siglo i a.C. (Roldán, 1968; Mangas y Solana, 1985, 12-24; Francisco, 1989). Parece que la inestabilidad en la zona puede darse por concluida con la llegada al poder de Augusto, poco antes de la pacificación de toda la Península a través de las guerras cántabro-astures. En este contexto la constitución de la nueva provincia Hispania Vlterior Lusitania y el establecimiento de su capital en Augusta Emerita se enmarcan dentro de una serie de medidas destinadas al sometimiento definitivo de estos pueblos y la conquista definitiva de toda la Península (Sayas, 1979, 744 ss).

No existe una opinión unánime sobre la forma en que se hace efectiva la división de la Ulterior ni sobre el momento concreto en que surge Lusitania como provincia. Las opiniones de los autores se dividen al respecto:

- Para algunos autores, la división de la Hispania Ulterior en dos provincias - Bética, que queda en manos del senado, y Lusitania, bajo control imperial-y la transferencia de los nuevos territorios con- 


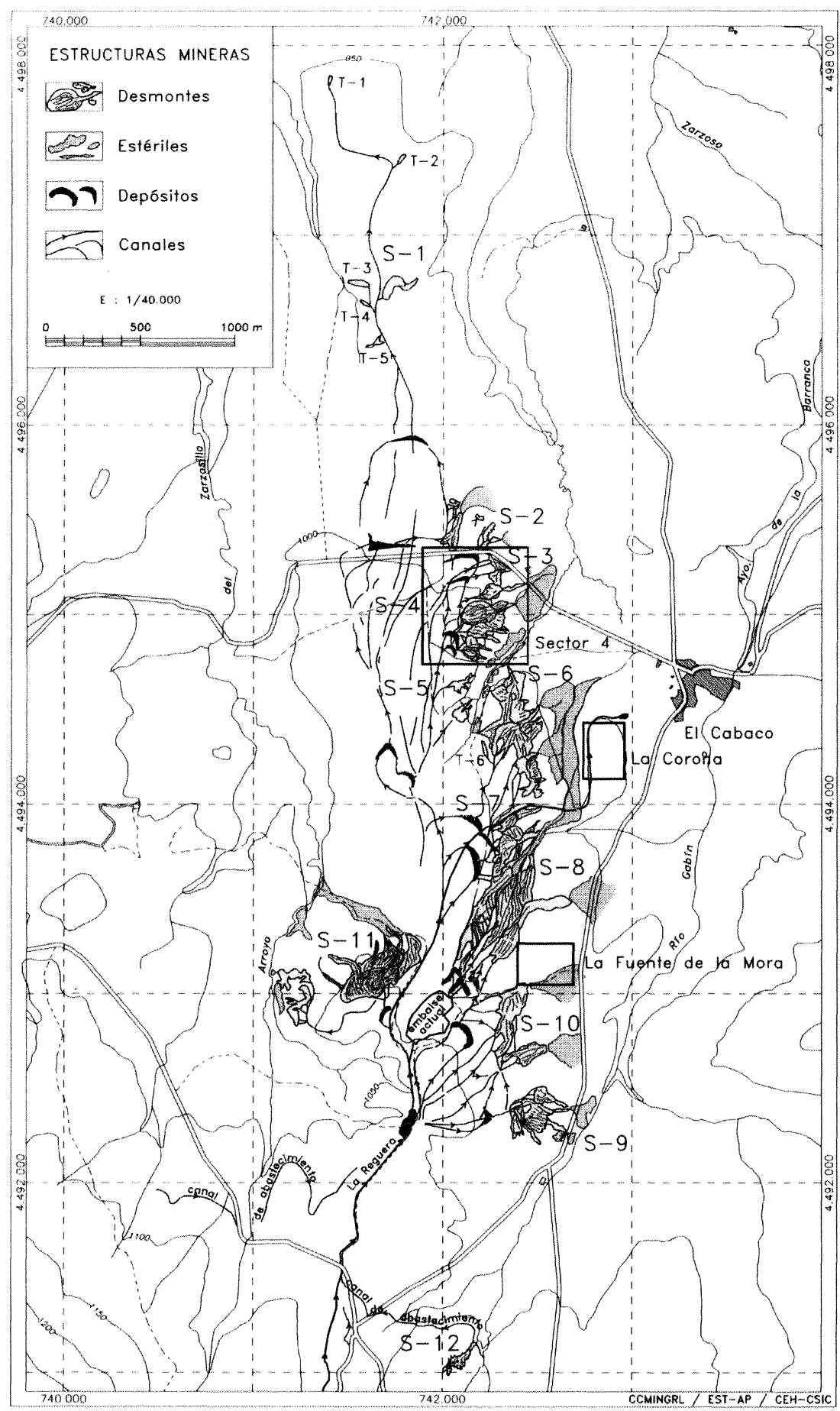

Fig. 3.-Estructuras mineras de Las Cavenes de El Cabaco a partir de la fotointerpretación aérea.

quistados, Asturia y Callaecia, a la Citerior, se habría realizado en un único momento, antes del final del reinado de Augusto. La fecha concreta de esta reorganización todavía sigue siendo discutida.
- Otros autores consideran que la división en tres provincias se realizó en varias etapas sucesivas. En un primer momento Augusto dividiría la Ulterior en dos provincias, adscribiendo a la Lusitania los terri- 
torios del noroeste peninsular aún sin pacificar. Esta primera reorganización respondería fundamentalmente a motivaciones estratégicas y sería coincidente con el comienzo de las operaciones contra cántabros y astures, en torno al 27 a.C., que es la fecha transmitida por Dion Casio (LIII, 12,4). Una vez pacificados los territorios del noroeste, Augusto reorganizaría de nuevo las fronteras - delimitación que perdura durante todo el alto imperio - adscribiendo a la Citerior los territorios de astures y galaicos así como la parte oriental de la Sierra Morena (la región de Castulo). La datación de esta segunda reorganización plantea también varios problemas. Algunos autores aceptan la fecha proporcionada por Dion $\mathrm{Ca}$ sio, en torno al 14 a.C., que coincide con la segunda estancia de Augusto en Hispania; otros tienden a situarla en torno a los años $7 / 2$ a.C. ${ }^{6}$

El nacimiento de la provincia de Lusitania y los reajustes de fronteras efectuados en los territorios de las provincias hispanas se han puesto en relación con razones de índole estratégica y militar relativas a la zona del noroeste peninsular. Según algunos autores el hecho de que, al acabar las guerras, se reajusten de nuevo los límites de las provincias adscribiendo los territorios de astures y galaicos a la Citerior, no haría sino confirmar que las motivaciones de esta reorganización fueron fundamentalmente de orden político-militar, sin que existan motivaciones económicas relevantes (Francisco, 1989, 93; Domergue, 1990, 200). Es difícil determinar en qué medida los factores militares han intervenido para que desde el principio se efectuara una división entre las provincias de Bética y Lusitania, pero es cierto que no fueron las únicas razones de peso. Otra serie de circunstancias debieron jugar un papel más importante para que en fechas sucesivas se fueran fijando los límites entre la Bética y la Citerior, la separación entre Lusitania y Bética y la división entre las provincias Lusitania y Citerior. Podemos observar ya en las medidas reorganizativas llevadas a cabo en estos años un interés especial del estado romano por los recursos de las regiones recién incorporadas y reorganizadas, entre los cuales el oro, como recurso fundamental dentro de la nueva política de Augusto, va a jugar un papel de primer orden (D.S. 52, 48, 4-5 y Flor. 2, 33, 60).

La falta de articulación que presentan los territorios de galaicos y astures antes del dominio romano hizo que el periodo bélico en el Noroeste, después de la finalización de las guerras, se prolongara hasta los años centrales del siglo ı d.C. La larga dura-

${ }^{6}$ Sobre el debate en torno a este tema: Sayas, 1979; Francisco, 1989; Domergue, 1990. ción de las guerras cántabro-astures parece transmitir un costoso control de la zona que se ve sometida a una profunda reorganización para su completa integración en el imperio, basada en el afianzamiento de los elementos de control y articulación esbozados durante las campañas militares (Orejas y Sánchez-Palencia, e.p.). En los territorios del norte de Lusitania recién pacificados las transformaciones operadas durante el siglo I a.C. habían sido lo bastante sensibles para que la reorganización administrativa se efectuara de forma diferente a la del norte ${ }^{7}$. Así, al separarse Lusitania tanto de la Bética como de Asturia y Callaecia «quedaba constituida en la parte occidental de la Península una zona entre el Duero y el Guadiana más o menos homogénea, que había experimentado ya transformaciones sensibles no resaltadas debidamente» (Sayas, 1979, 746). De esta forma, con la adscripción tras la conclusión de la conquista de los nuevos territorios a dos provincias imperiales diferentes, Citerior y Lusitania, Augusto se garantizaba no sólo el control efectivo sobre las dos zonas, sino también sobre la explotación de sus recursos. Igualmente, los intereses económicos debieron pesar bastante en la transferencia, en este mismo momento, de la zona minera de Castulo a la Citerior, dejando en la Ulterior la región oriental de la Sierra Morena ${ }^{8}$.

2. El papel de la minería en El CONTEXTo de la REESTRUCTURACIÓN TERRITORIAL DEL NORDESTE DE LUSITANIA

\subsection{La reorganización regional}

Las transformaciones experimentadas en los territorios de lusitanos y vettones en los años anteriores al cambio de era quedaron refrendadas con las

7 A lo largo del siglo I a.C. los principales núcleos urbanos vettones parecen haber experimentado un desarrollo notable desde sus estructuras prerromanas, quizás al amparo de una cierta marginalidad respecto a los escenarios bélicos o más conflictivos. El mejor testimonio de esto lo constituiría la pervivencia de los principales oppida vettones hasta época romana (Salinas, 1990 y 1992-93; Edmondson, 1992-93; Álvarez, 1999).

8 Domergue ve en la incorporación de la zona minera de Castulo a la Citerior motivos exclusivamente estratégicos, como el reforzar la seguridad en una zona de frontera, conflictiva, con lo que se estaría buscando el proteger la recién creada via Augusta entre Cartagena y el valle del Guadalquivir. Sin embargo, en esta época, la presencia romana en la zona ya estaba muy consolidada y la explotación de las minas corría a cargo de societates publicanorum. Con la anexión a la Tarraconense, la región y todos sus recursos pasan a estar bajo control directo del emperador. Sobre la importancia de los recursos mineros en esta zona fronteriza y su evolución desde época ibérica: Chapa y Mayoral, 1998. 
medidas destinadas a la integración de la nueva provincia en los marcos administrativos del imperio. Tras la reorganización Lusitania sigue bajo control imperial, pero no se conservan tropas en sus territorios. Algunos autores han defendido la posible existencia de un asentamiento en la ciuitas Igaeditanorum del Ala I Singularium ciuium Romanorum (Francisco,1989, 156 ss y 167-169) a partir de dos inscripciones ${ }^{9}$ aparecidas en los territorios adscritos a esa ciuitas. Tanto Roldán como Le Roux piensan que la mención de dos personajes pertenecientes a esta unidad no es suficiente para realizar esta afirmación y que no existen pruebas concluyentes para defender su presencia en Lusitania, que de admitirse, debió ser corta, pues se encuentra en Germania en el 68/69 d.C. (Roldán, 1974; Le Roux, 1982).

La vinculación establecida desde el principio entre los territorios recién incorporados, caracterizados por distintos grados de integración que se reflejan en un planteamiento diverso de la reorganización por parte de la administración romana, se refuerza con la creación de la calzada de La Plata, que se convierte con la nueva división provincial, y el establecimiento de Augusta Emerita como capital en el eje principal de conexión de la provincia y en vía de unión entre los nuevos territorios conquistados, tomando así un carácter administrativo importante y un papel articulador de primer orden entre los territorios lusitanos y de astures y galaicos. La misma fundación de Augusta Emerita tiene un papel decisivo en la consolidación de la presencia romana en la zona. La nueva capital constituye un eje de unión entre las regiones meridionales y los territorios del norte. Cuando en época de Augusto se establece el campamento de Astorga (González, 1999, 95), la vía norte-sur gana en importancia quedando establecida como camino unitario de Emerita hasta Asturica ${ }^{10}$.

Con la reorganización de las fronteras y el establecimiento de los puntos fundamentales de una nueva estructura administrativa — articulación del territorio a partir del reforzamiento de las vías principales, fundación de Emerita - se irá imponiendo

${ }^{9}$ HAE, 1077 y 1078 . Francisco menciona además la existencia de otro eques alae primae en Caurium (1989, 156, nota 296) que en su opinión apoya la presencia de esta unidad en la zona.

10 A pesar de este carácter unitario de la calzada hay algunos elementos que señalan que su configuración cambia con el cambio de frontera, al pasar de Lusitania a la Citerior: ausencia de miliarios en el tramo de la Citerior, desarrollo diferente de la calzada, etc. Salamanca parece ser el punto de conexión y al mismo tiempo de ruptura de la misma, cuando el verdadero punto de conexión entre los tramos procedentes de Mérida y Astorga se encontraría en Zamora (Roldán, 1971,21 y 168 ss). una nueva orientación económica de acuerdo con los intereses imperialistas del estado romano. Es a partir de este momento cuando se observa un primer esfuerzo de homogeneización y organización que afecta a todos los territorios provinciales, centrado fundamentalmente en aquéllos recién conquistados en los que el estado tiene un interés particular. Este interés se refleja de manera especial en la intensa reestructuración a la que fueron sometidos tras la reorganización de las provincias los territorios del norte de Lusitania, reorganización que tiene su mayor testimonio en la serie de termini augustales repartidos en los territorios situados entre el Tajo y el Duero.

De la provincia de Salamanca proceden cuatro epígrafes que conciernen de forma directa a la región que estamos estudiando: de Ledesma procede un trifinium (CIL II, 859), que separaba los territorios de Bletisa, Mirobriga y Salmantica; de Ciudad Rodrigo proceden dos hitos (CIL II, 857 y 858) que delimitaban los territorios de Bletisa, Mirobriga, Salmantica y Valuta (?); un cuarto hito terminal se halló en Yecla de Yeltes (CIL II, 5033), testimoniando el límite entre los Mirobrigenses y los [...]polibedenses ${ }^{11}$. De Portugal proceden otros tres epígrafes. El único que permite conocer el nombre de las comunidades afectadas por la delimitación es el que procede de las proximidades de Idanha, entre Monsanto y Valverde (CIL II, 460), que separaba los territorios de Lancienses Oppidani e Igaeditani; de Oliveira de Azemeis procede un hito terminal (HAEp 1442) que delimitaría las comunidades de los Talabrigenses (?) y Lancobrigenses (?); de Guardão procede un tercer epígrafe $(A E 1954,88)$ que no permite conocer el nombre de la comunidad implicada [...]ienses. Todos los epígrafes se han fechado entre los años 2 a.C.-14 d.C., pero es destacable que los que incluyen datación se fechan entre los años 5/6 d.C. ${ }^{12}$ Nos hallamos ante una operación de reorganización territorial de gran envergadura, que afecta con seguridad a toda Lusitania al norte del Tajo y que la mayor parte de los autores está de acuerdo en relacionar con la reorganización general de las provincias efectuada por Augusto y el establecimiento definitivo de la frontera lusitana en la línea del Tormes (Edmonson,

1 Sobre la identificación de las comunidades de Valuta (?) y Polibeda (?) y la interpretación de los epígrafes que las mencionan: Mangas, 1992, 256-266.

12 Junto a estos epígrafes augusteos hay que mencionar un cuarto epígrafe procedente de Goujoim (Armamar, Viseu), que delimitaba el territorio de los Coilarni y los Arabrigenses (?) ( $A E$ 1979, 331), fechado en el 46/47 o 59 d.C. Los termini lusitanos están recogidos por: Edmonson, 1990a y 1990b, 124; Le Roux, 1994, 40; Ariño y Rodríguez, 1997. 
1990a y 1992-93, 27; Salinas, 1990, 260 y 1992, 305; Ariño y Rodríguez, 1997, 229).

Conseguida la pacificación de los nuevos territorios Roma se plantea la necesidad del control de una serie de espacios extensos y diversificados, dentro de los cuales se encuentran numerosas comunidades peregrinas que hasta ese momento habían escapado a la obligación de un inventario de sus bienes y a la definición precisa de sus límites. Sólo a comienzos del Principado se hace patente por primera vez un esfuerzo de delimitación y organización sistemática de los territorios provinciales. Es en ese momento cuando se ponen en marcha intervenciones sistemáticas para la regulación del suelo provincial como el ager per extremitatem mensura comprehensus transmitido por Frontino ${ }^{13}$. La mayoría de los autores ha puesto en relación este tipo de ager con la serie de termini lusitanos conocidos, pero vinculándolo a cuestiones de carácter local en el marco de las relaciones de propiedad en el interior de las comunidades implicadas. Así, el ager per extremitatem se ha interpretado en relación con la existencia de campos que serían propiedad comunal de las organizaciones gentilicias de los pueblos del norte de la meseta (Vigil, 1973) o como un tipo agrimensorio adscribible a terrenos públicos en el interior del territorio de las ciudades (Salinas, 1989, 107 y 1992-93, 184).

En un artículo reciente, Orejas y Sastre proponen la interpretación de toda esta serie de epígrafes relacionados con la fijación de límites del territorio de ciuitates con la definición de esta forma de organizar el suelo peregrino y tributario transmitida por Frontino, en el marco de la ordenación territorial a la que fueron sometidas las comunidades del Noroeste peninsular. Tras la conquista, Augusto procedería a la organización de las comunidades imponiendo el sistema de ciuitates, sobre las que se construye el nuevo sistema territorial impuesto; tras la nueva definición provincial «Rome a procédé à la délimitation de ces nouvelles entités territoriales moyennant l'assignation aux communautés d'un territoire uniuersus modus, c'est-à-dire, grâce à la formule gromatique de l'ager per extremitatem mensura comprehensus» (Orejas y Sastre, 1999, 172). Como las autoras subrayan, este tipo de organización debió ser aplicada de forma general, con sus implicaciones fiscales y censuales, en la reorganización del noroeste por Augusto de manera más frecuente de lo que se ha supuesto; el hecho de que los ejemplos seleccionados por Frontino, Palantia y Salmantica, pertenezcan a Hispania, a las provincias Citerior y Lusitania recién reorganizadas, indica que esta solución pudo

13 De Agrorum qualitate, $7-9=$ Th. 1-2. tener importancia en las intervenciones de Augusto en términos generales (Orejas y Sastre, 1999) ${ }^{14}$. La unidad básica sobre la que se construye el sistema provincial es la ciuitas: Roma toma como referencia de la imposición tributaria a la comunidad, a la que dota de independencia a la hora de repartir las cargas internamente. Como señalan las autoras, en los territorios del Noroeste la intervención administrativa romana fue especialmente intensa, ya que conllevaba la consolidación de nuevas formas de organización radicalmente distintas a las de las comunidades castreñas prerromanas (Orejas y Sastre, 1999; Sastre, 1998). En el caso de los territorios del norte de Lusitania parece que la delimitación de las nuevas entidades territoriales se articuló en torno a algunos núcleos que durante los años previos al cambio de era parecen haber adquirido una cierta relevancia con respecto a otros del territorio circundante ${ }^{15}$.

\subsection{El desarrollo de la minería y la explotación de nuevos recursos}

La reorganización de las fronteras provinciales, el reajuste del territorio de Augusta Emerita y la delimitación en ciuitates de los territorios situados al norte del Tajo muestran la profunda intervención

14 En relación con los termini augustales lusitanos y el texto de Frontino se puede poner también un mojón de carácter técnico procedente de Villamiel (Cáceres) en el que se leen los nombres de las comunidades Vinia Campegiensis y Valseni (Ariño y García de Figuerola, 1993; Ariño y Rodríguez, 1997). En el artículo citado de Orejas y Sastre se recogen otra serie de documentos que apuntan en esta misma dirección: los hitos terminales que separaban los territorios de ciertas ciuitates astures de los de prata y otras referencias a una amplia serie de textos procedentes de otros territorios que se pueden interpretar en el mismo sentido (Orejas y Sastre, 1999, notas 20-23).

is Sobre los núcleos citados en los termini de la provincia de Salamanca y su desarrollo en época romana: Le Roux, 1990, 46-47; Edmonson, 1990; Santonja, 1991, 26-27; Mangas, 1992. Los datos procedentes de las excavaciones realizadas en Salamanca han sido sintetizados por N. Benet en $\mathrm{Nu}$ mancia 6 (1996), 339 y 7 (1999), 281-83; ver también Martín Valls, R.; Benet, N.; Macarro Alcalde, C., 1991: Arqueología de Salamanca. Del Paleolítico a la Historia, Salamanca, 137-163. Sobre la posible evolución de Salmantica a municipio: Salinas, 1990, 260-61 y 1992; Francisco, 1993. Sobre Ciudad Rodrigo: Martín Valls, R., 1965: Investigaciones arqueológicas en Ciudad Rodrigo. Zephyrus, 81-98; idem, 1976: Nuevos hallazgos arqueológicos en Ciudad Rodrigo. Zephyrus, 373-388; Pradales Ciprés, D., 1992: Nuevos hallazgos cerámicos de TSH en la provincia de Salamanca. Actas I Congreso de Historia de Salamanca. Salamanca, T. I, 323 ss.; Numancia, 4 (1993), 337. Las excavaciones realizadas en Ledesma han puesto en evidencia un poblamiento continuado desde el Bronce final: Benet, N.; Jiménez, M.C.; Rodríguez, M.B., 1991: Arqueología en Ledesma, una primera aproximación: la excavación en la Plaza de San Martín. Del Paleolítico a la Historia. Salamanca, 111-136. 
del Estado romano y la voluntad de organización y homogeneización de estos territorios con vistas a su plena integración en el imperio. El hecho de que esta reorganización no diera lugar a una estructuración de orden municipal no puede de ninguna manera llevarnos a afirmar que en esta zona las medidas «tendentes a la integración de los hispanos en las estructuras institucionales del imperio debieron tener muy escasa repercusión» (Salinas, 1986, 40). Es precisamente a partir de este momento cuando se comienzan a sentir los efectos de la nueva orientación dada por Augusto a estos territorios. La fuerte reorganización de la región afectó de forma global a los recursos y poblaciones de la zona. Aunque no es mucho lo que sabemos acerca de la organización del poblamiento del suroeste de la provincia, lo que sí es seguro es que su articulación no se puede aislar del desarrollo de la explotación sistemática de los recursos, entre los cuales las minas debieron jugar un papel importante.

A comienzos del siglo I d.C. se pone en marcha la explotación a gran escala de los yacimientos auríferos de Lusitania, de forma contemporánea a los del Noroeste. Los trabajos realizados en El Cabaco muestran que las explotaciones de Las Cavenes estaban siendo puestas en explotación ya en los años centrales del siglo I d.C. ${ }^{16}$. Es en este momento cuando se ponen en valor de forma organizada los yacimientos auríferos del noroeste de la Península: el desarrollo de la actividad minera a partir del final del reinado de Augusto se realizaría al mismo tiempo en las tres grandes regiones auríferas - Lusitania, Callaecia y Asturia- que parecen constituir un todo a los ojos de la administración romana. En este sentido se puede interpretar la cita de Plinio acerca de las cifras de producción de las minas de oro del noroeste (Plin. NH, 33, 78). Por encima de la gestión provincial de la explotación de las minas, existe un control conjunto y directo del estado sobre el total de la producción. Ya en este momento la minería de oro era patrimonio exclusivo del estado y, como en el Noroeste peninsular, las minas lusitanas debieron estar gestionadas directamente por la administración romana.

Las transformaciones efectuadas en las estructuras territoriales de esta región muestran el interés específico de Roma por la zona dentro de la provincia. Este especial interés se manifiesta en la aparición de una serie de procuratores Lusitaniae et Vettoniae y de cargos subalternos dependientes de

${ }^{16}$ Los sondeos realizados en la Fuente de la Mora han proporcionado abundante material cerámico con una cronología situada entre el 30 d.C. - finales del siglo II d.C. ellos a partir del siglo II d.C. ${ }^{17}$. Según algunos autores la aparición de cargos con una adscripción territorial específica a Lusitania y Vettonia estaría testimoniando la existencia de dos distritos financieros independientes creados en un cierto momento por la administración romana, para facilitar la gestión dentro de la provincia (Roldán, 1968, 98). Los datos que poseemos no permiten afirmar la existencia efectiva de una división administrativa de Lusitania en un determinado momento, pero en este sentido nos parece interesante recordar la existencia de cargos en el Noroeste con una adscripción específica a Asturia et Callaecia y que han sido interpretados en el contexto de las soluciones que el estado romano adopta en relación con las necesidades surgidas de la explotación del oro. El estado subraya de esta forma la singularidad del Noroeste dentro de la Citerior reforzando su control con la creación de una organización administrativa específica (Sánchez-Palencia y Orejas, 1994, 172 ss).

La reorganización territorial debió contemplar, además de la delimitación territorial de las comunidades en una serie de ciuitates, la aparición de otras unidades territoriales que pudieron estar relacionadas con la existencia de extensas áreas ocupadas por las explotaciones auríferas y que formaban parte del ager publicus (Sánchez-Palencia y Orejas, 1994, 176). Como veremos, los rasgos de la región no se pueden aislar del desarrollo de la actividad minera y sólo desde una perspectiva global podemos entender el alcance que ésta tuvo en las transformaciones de las estructuras sociales y territoriales de la zona y en la puesta en marcha y revalorización de los recursos. Las minas de oro implican una infraestructura de ordenación territorial que supera el marco estrictamente local: la explotación se basó en una labor organizadora a gran escala que afectó al conjunto del poblamiento y a la ordenación de los recursos, por lo que su estudio no se puede abordar aisladamente, sobre todo desde el momento en que aparecen asociados ${ }^{18}$.

En la zona minera de Las Cavenes hemos podido individualizar la existencia de espacios dedicados a la explotación agropecuaria localizados entre grupos de labores, formando parte de la articulación general del conjunto de la explotación. Parte de

17 CIL II, 484, 1178, 1267; VI, 31856; y un tabularius prouinciae Lusitaniae et Vettoniae (CIL II, 485). La transcripción de los epígrafes y una discusión acerca del significado de estos documentos en Roldán, 1968.

${ }^{18}$ Los trabajos realizados en zonas mineras del Noroeste han mostrado que algunos de los nuevos asentamientos se encuentran físicamente integrados en las labores mineras (Sánchez-Palencia y Orejas, 1994; Orejas, 1996; SánchezPalencia y otros, 1996). 


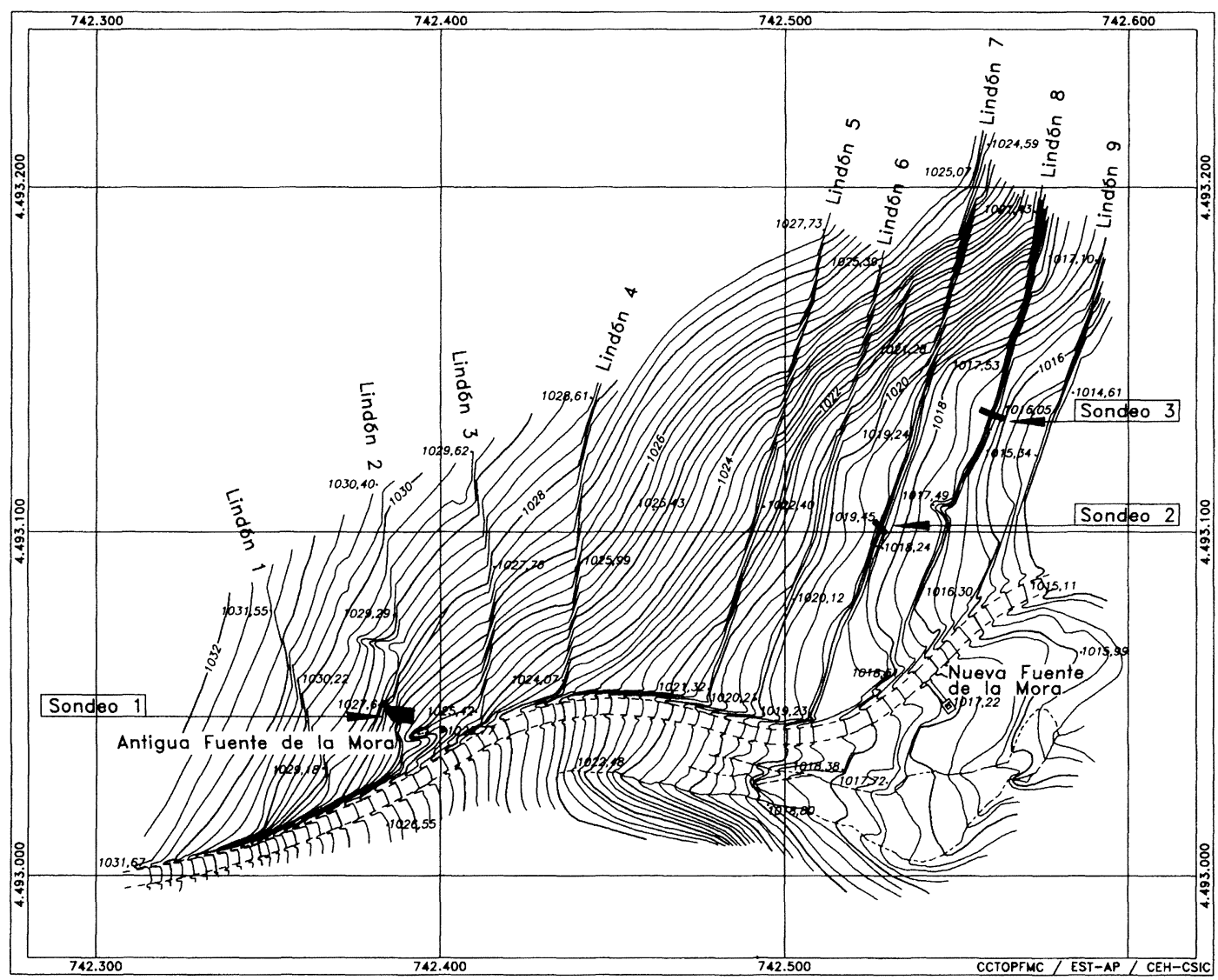

Fig. 4.-Plano topográfico del Sector de la Fuente de La Mora con la situación de «los lindones» y de los sondeos realizados.

nuestros trabajos se han centrado en un lugar, La Fuente de la Mora, situado entre dos de las principales labores, los sectores de explotación S-10 y S8 , donde se puede reconocer sobre el terreno la presencia de una serie de bancales que, a modo de terrazas, se alinean a lo largo de la ladera ocupando una amplia extensión (figs. 3 y 4). El interés de este lugar por su situación en plena explotación minera y la ausencia de indicios en superficie nos llevaron a plantear varios sondeos que han permitido documentar la naturaleza de estos aterrazamientos ${ }^{19}$.

${ }^{19}$ El lugar es mencionado por Morán $(1946,29)$ y Maluquer $(1956,53)$ (ver nota 2). La Fuente de la Mora forma parte de las leyendas populares de la zona que hablan de la existencia de restos antiguos en este lugar. Los habitantes del Cabaco ya mencionaban la existencia de algunos «lindones» y la aparición en uno de ellos de restos de cal y tejas. La zona se encuentra en la actualidad ocupada por un bosque de roble y no se recuerda que haya sido cultivada, al menos, en los últimos cien años. El bosque y la abundante vegetación de monte bajo (brezo, jara, helecho) impiden la visibilidad de cualquier tipo de evidencia en superficie, por lo que lo único que se reconoce sobre el terreno son estos aterrazamientos.
Cada uno de los tres sondeos realizados cortó longitudinalmente una terraza en un tramo de su recorrido de forma que en los tres casos pudimos documentar en sección la estructura del bancal (figs. 4-7). Las unidades estratigráficas identificadas en los perfiles de los sondeos se corresponden en la mayoría de los casos con unidades de suelo distintas, por lo que su denominación y registro se llevó a cabo atendiendo a un doble criterio: por una parte, en tanto que unidades arqueológicas diferentes, cada una de las unidades reconocidas en el perfil se contemplan como unidades estratigráficas (UE) distintas. Junto a la numeración en UE, los distintos suelos identificados se han designado con las siglas edafológicas de horizontes, siguiendo las normas generales de denominación de horizontes (Porta y otros, 1994) ${ }^{20}$.

${ }^{20}$ Los horizontes edafológicos se designan entre paréntesis por medio de letras mayúsculas que traducen la información de cada uno de ellos, indicando su posición en el suelo, el proceso genético preponderante en su formación y la característica o propiedad más destacable dentro del mismo. 
Las secuencias estratigráficas documentadas en los tres sondeos presentan características similares. Las variaciones en estas secuencias se deben más a los procesos a los que se han visto sometidas las terrazas tras su abandono que a diferencias constructivas importantes. Como se observa en la figura 5 , en el primero de los sondeos, bajo las UE 1 y 2 $\left(\mathrm{O}_{1}\right.$ y $\left.\mathrm{O}_{2}\right)$ se encuentra la UE 3 , un horizonte $\mathrm{O}_{3}$ formado por los aportes de materia orgánica y por el arrastre del material erosionado situado ladera arriba, como demuestra la gran cantidad de fragmentos de cerámica y teja romana procedente de la excavación en planta de este nivel. La UE 4 no tiene designación de horizonte, puesto que es la unidad que interpretamos como el muro de contención construido para retener el suelo destinado al cultivo. Aunque esta unidad se encuentra prácticamente desmantelada, su estructura ha condicionado de manera particular la forma en que se han dispuesto los horizontes formados tras el abandono de la zona. La UE 5 $\left(A b_{1}\right)$, contemporánea del funcionamiento del muro de contención (UE 4), es el suelo que interpretamos como activo y dedicado al cultivo en época romana; en él aparecieron abundantes fragmentos de cerámica común, teja y terra sigillata. Los procesos desarrollados en este horizonte han provocado su desplazamiento hacia el lado este del corte, invadiendo parcialmente la UE $6\left(\mathrm{Ab}_{2}\right)$. Este nivel es un horizonte enterrado que pudo estar afectado también por actividades de laboreo. Podría tratarse de la superficie de cultivo del bancal situado en el nivel inmediatamente inferior al que estamos estudiando; esta hipótesis vendría confirmada por la existencia de abundantes puntos de materia orgánica visibles en

$\overline{\text { Con la letra «O }} »$ se denominan los horizontes orgánicos formados en la parte superior del suelo en condiciones predominantemente aerobias. Estos suelos contienen un $20 \%$ o más de carbono orgánico. Es el horizonte típico de los suelos de bosque. Con la letra «A» se designan de forma genérica aquellos horizontes minerales oscurecidos por aportes de materia orgánica. También se designa como A cualquier horizonte afectado por laboreo o pastoreo. Si este horizonte se halla en superficie se caracteriza con las letras «Ap»; en nuestro caso hemos optado por la denominación «Ab» para significar que se halla enterrado. Los horizontes «B» son horizontes minerales situados en el interior de un suelo, bajo un horizonte «A». Con la letra «C» se designa un horizonte mineral que en comparación con los anteriores se encuentra poco afectado por los procesos edafogénicos. En todos los casos los índices numéricos que acompañan a la denominación del horizonte sirven para designar una secuencia en la posición del horizonte dentro del suelo. Para el estudio de horizontes genéticos en edafología son de gran utilidad los trabajos de Bonneau y Souchier, 1987; Gandullo, 1984. Por la utilización de los horizontes edafológicos para el estudio de perfiles arqueológicos son muy interesantes los trabajos de Butzer (1976 y 1989) y las síntesis generales de Chouquer y Favory (1991, 42-46 en especial, con bibliografía más amplia). este estrato y por la composición de la tierra de ambos niveles ( 5 y 6 ), que es muy similar. Estas dos UE se asientan sobre la UE 7 (B), un horizonte mineral con abundantes manchas de materia orgánica que interpretamos como el primer suelo existente en la zona sobre el substrato natural, y que fue integrado parcialmente en los horizontes $\mathrm{Ab}$ al asentarse la estructura de bancales. Esta última UE se asienta directamente sobre la UE 8 (C), el substrato original de la zona.

Una secuencia similar se comprobó en el segundo de los sondeos realizados, situado a menor altura en la ladera: UE $1\left(\mathrm{O}_{1}\right)$ - UE $2\left(\mathrm{O}_{2}\right)$ - UE 3 - UE $4(\mathrm{Ab})$ - UE $5(\mathrm{C})$ (fig. 6) ${ }^{21}$.

La tercera de las terrazas presenta unas condiciones de conservación bastante buenas. Como se puede observar en su perfil oeste (fig. 7), bajo los horizontes orgánicos $\left(\mathrm{O}_{1}\right.$ y $\left.\mathrm{O}_{2}\right)$ se localiza la UE 4, a la que como en los anteriores cortes no hemos atribuido la designación de horizonte ya que consideramos que se trata del bancal propiamente dicho. Esta estructura sirve de contención al suelo trabajado, identificado en este corte en la UE 3 (Ab). El bloque de granito que se sitúa directamente sobre el sustrato natural pudo formar parte de la estructura de contención del bancal, al igual que formaban parte de esta estructura algunos fragmentos de teja romana y de cerámica común, muy poco rodados, que documentamos en el perfil al realizar el corte. La estructura del bancal (UE 4) y la UE 3 están sellando dos bolsadas de tierra muy compacta a las que hemos denominado $\mathrm{B}_{1}$ y $\mathrm{B}_{2}$ (UE 5 y 6). Consideramos que estas UE son los restos conservados del suelo anterior a la construcción del bancal, que fue integrado en el nuevo horizonte $A$, formado una vez que esta zona se puso en cultivo. Ambas unidades se asientan sobre el sustrato natural de la zona, la UE 7 (C).

En los tres casos la existencia del muro de contención de la terraza ha condicionado la forma en que se han dispuesto las unidades estratigráficas formadas tras el abandono de la explotación de esta zona, favoreciendo la aparición de una morfología característica, los bancales o «lindones», fosilizada en el paisaje actual. El hecho de que se trate de una zona de bosque, que no ha sido dedicada a una explotación agropecuaria, ha permitido que la estructura original de los bancales se haya conservado y se refleje en una serie de rasgos fácilmente observables tanto en la superficie como en los perfiles an-

${ }^{21}$ En este caso se realizó una limpieza superficial de la terraza que permitió documentar la estructura que presentan los bancales en la actualidad. La limpieza del muro de contención proporcionó varios fragmentos de teja y cerámica común romana, muy rodados, y algunas escorias. 

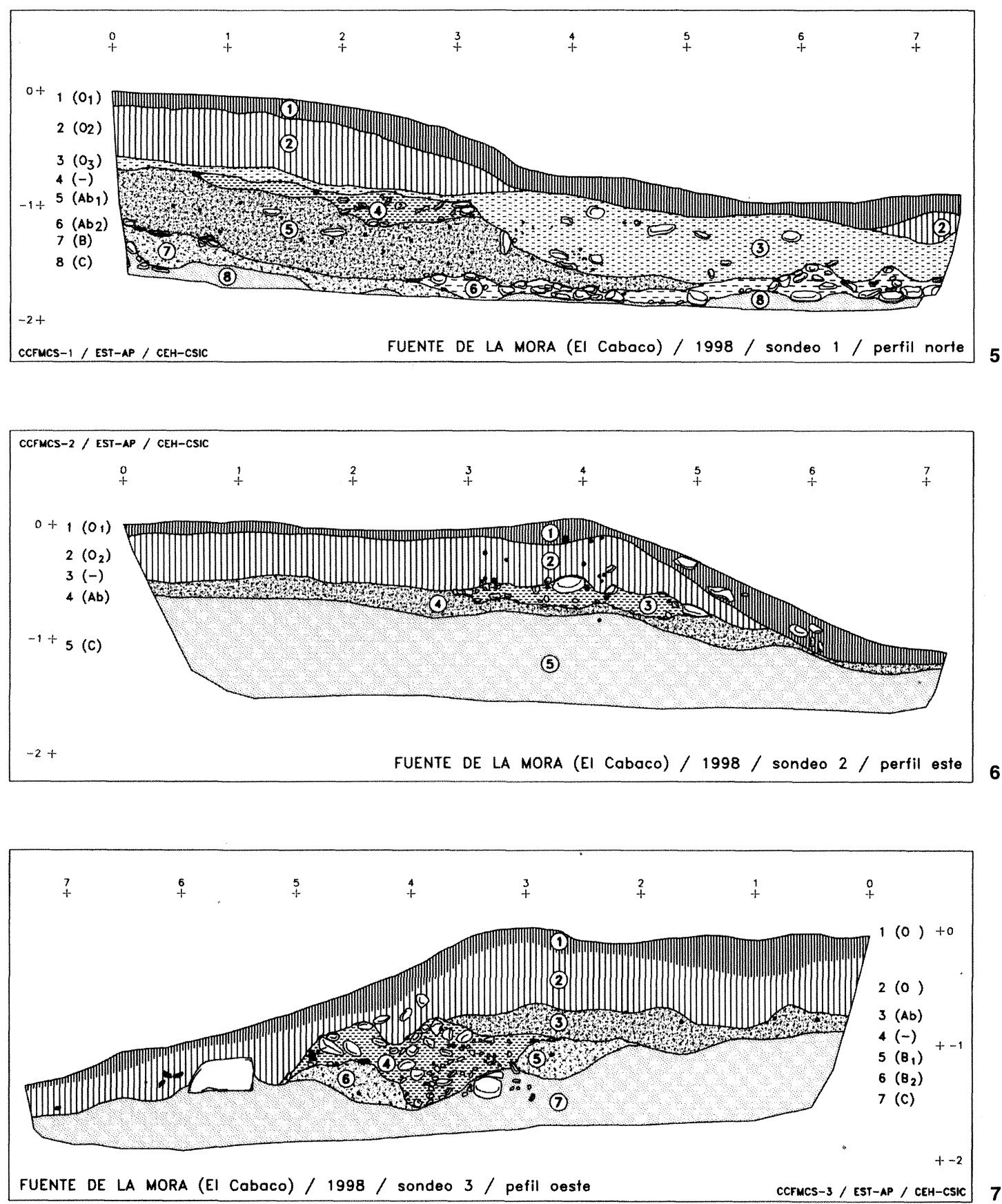

Figs. 5, 6 y 7.-Perfiles estratigráficos de los sondeos 1,2 y 3 de la Fuente de la Mora, con indicación de la correspondencia entre unidades estratigráficas y horizontes edafológicos.

teriormente descritos. El muro de contención que forma el aterrazamiento se documenta claramente en los tres cortes realizados. Su estructura constructiva es especialmente clara en el perfil del tercer sondeo: está formado por una acumulación de cantos rodados de buen tamaño, con la inclusión a ve- ces de fragmentos de tégula y cerámica común, trabados con tierra compactada.

La morfología exterior de los bancales es uno de los indicadores más evidentes de la existencia y funcionalidad de este tipo de estructuras relacionadas con la explotación agraria, pero no es el único dato 
que nos informa acerca las condiciones bajo las que se han formado estos aterrazamientos. Dada la complejidad de los factores que intervienen en la formación de los suelos de un determinado territorio, no se pueden definir criterios inequívocos que puedan ser usados para el estudio de las prácticas agrarias, pero otra serie de características morfológicas nos permiten inferir con un cierto grado de fiabilidad las actividades realizadas en la zona que estamos estudiando; los efectos del arado y del laboreo de las tierras de un territorio, así como sucede con los de otras actividades como la explotación minera, poseen sus propias manifestaciones morfológicas, ya que crean unas condiciones específicas en el terreno sobre las que se desarrollarán los procesos de formación del paisaje posteriores.

Una de estas manifestaciones es la aparición en los perfiles de un estrato homogéneo (como la UE 3 del tercer sondeo), no necesariamente espeso, en la parte superior de un suelo enterrado, sobre el que se han formado varios horizontes orgánicos (UE 2 y UE 1 en ese mismo sondeo). Este tipo de estratos son un indicador claro de un laboreo pasado. Cuando un suelo es arado y puesto en cultivo se forma en su superficie lo que se llama la «suela de labor», una especie de costra superficial muy compacta que impide la erosión de la superficie del suelo al protegerlo de la lluvia o de la acción de otros procesos exteriores que pueden producir su degradación y empobrecimiento. Cuando un suelo que ha sido cultivado se abandona y deja de ser trabajado, este nivel más duro que se ha formado en su parte superior impide la asimilación de nuevos componentes, favoreciendo así la formación por acumulación sobre el antiguo suelo de nuevos horizontes que lo van enterrando progresivamente (Butzer, 1989, 122ss). En el perfil la formación de un horizonte orgánico sobre un suelo cultivado con suela de labor se caracteriza porque el límite que separa ambos horizontes es abrupto, es decir, la distancia vertical a lo largo de la cual se produce el cambio de horizontes es pequeña, menor de $2,5 \mathrm{~cm}^{22}$.

Todo lo dicho anteriormente no quiere decir que los estratos cultivados se conserven bajo cualquier circunstancia; pueden haber sido modificados por la actividad humana posterior o por la acción de la vegetación natural antes de su enterramiento. Trabajos realizados en diferentes territorios (Courty y otros,

22 El límite abrupto producido por laboreo no es el único caso en el que se produce un límite nítido. Un límite de estas características puede indicar igualmente una superposición de materiales, es decir, una discontinuidad litológica. Así ocurre en el paso de la UE 7 a la UE 2 en el tercer sondeo.
1989) muestran que el cultivo de una tierra no ocasiona siempre un horizonte A caracterizado por una textura (la «suela de laboreo») determinada. Dependiendo de las propiedades del suelo y de la forma en que éste ha sido enterrado, sus características pueden perderse y los aportes posteriores, como la materia orgánica o los arrastres producidos por la erosión del terreno, pueden ser rápidamente integrados en su estructura. Si volvemos a los perfiles de los tres cortes podemos comprobar que en los tres casos los suelos asociados a los bancales se han conservado en mayor o menor grado. Únicamente en el tercero de ellos el suelo situado en la parte inferior del bancal, al sur del corte, y por tanto, sometido de forma más directa a la erosión al no encontrarse directamente vinculado a un elemento de protección como es el muro de contención del bancal, ha desaparecido, siendo asimilados sus componentes por el horizonte $\mathrm{O}_{2}$ representado por la UE 2 y que se asienta directamente sobre el sustrato natural.

La construcción de los bancales y la preparación para el laboreo de las tierras de la zona de la Fuente de la Mora supuso la incorporación, total o parcial, de los suelos preexistentes en los nuevos suelos de cultivo. Las labores de acondicionamiento del suelo no llegaron a eliminar por completo el suelo anterior, como demuestran en el tercer sondeo las bolsadas (UE 5 y 6) que se han conservado. Según se puede comprobar en los tres perfiles, uno de los primeros efectos de la puesta en cultivo de estas tierras fue la eliminación de los horizontes superiores preexistentes al laboreo, especialmente de los estratos ricos en materia orgánica, y la creación de un horizonte de cultivo homogéneo sostenido por el bancal. Este nuevo horizonte incluye, en principio, características del estrato húmico de superficie así como material mineral retrabajado proveniente del horizonte A preexistente ${ }^{23}$.

En la conservación de las características morfológicas producidas por la explotación agraria de esta zona influyen un gran número de parámetros relacionados con el tipo de suelo sobre el que se ha realizado el laboreo, la clase de cultivo que ha tenido lugar, la rapidez con la que los estratos cultivados fueron enterrados, etc. En el caso de la Fuente de la Mora son numerosos los rasgos morfológicos conservados que permiten la adscripción de los bancales a la explotación agropecuaria de la zona. Nos encon-

${ }^{23}$ Este tipo de perfiles, documentados particularmente en zonas agrícolas abandonadas, se denominan perfiles alterados; el perfil descrito en el tercer sondeo, en la parte inferior del bancal, en el que el horizonte $\mathrm{Ab}$ ha desaparecido por completo, se ha identificado y definido en otras zonas como perfil de suelo truncado (Butzer, 1989, 130). 
tramos ante suelos que han sido rápidamente enterrados (UE 3 en el tercer sondeo, UE 5 en el primero), por lo que se ha conservado otra serie de evidencias morfológicas que apoyan la hipótesis de la dedicación agrícola de estas estructuras. Nos estamos refiriendo a la topografía de algunos de los límites entre horizontes, en los que la forma de la superficie de separación entre éstos presenta unas características particulares. Así, el límite entre las UE 3 y UE 2 en el tercer sondeo, o entre la UE 6 y UE 3 en el primero, forma sinuosidades más anchas que profundas. La forma de estos límites podría estar asociada al laboreo con arado de estos suelos; los surcos habrían quedado enterrados en el subsuelo y fosilizados al igual que el horizonte al que pertenecen. Límites similares entre horizontes han sido reconocidos en varios estudios arqueológicos realizados en áreas rurales (Courty y otros, 1989) y utilizados como evidencia para documentar la existencia de caballones de cultivo asociados a suelos enterrados. En edafología este tipo de límites se han relacionado con el laboreo de los suelos y, en algunos casos, con la existencia de vías preferenciales de circulación de agua sobre éstos (Porta y otros, 1994) ${ }^{24}$.

En toda el área ocupada por las labores hay indicios de la existencia de bancales como los estudiados, en lugares vinculados a las estructuras de la minería romana. De momento sólo se puede afirmar esta relación para el caso de la Fuente de la Mora. Sobre la fotografía aérea se aprecia que el área ocupada por los bancales estudiados se articula con la red hidráulica de las labores por medio de un canal que, desde uno de los principales depósitos reguladores, desemboca en la cabecera de los aterrazamientos, abasteciendo así posiblemente la zona reservada a la explotación agraria (fig. 8).

\subsection{Las estructuras mineras de Las Cavenes}

El desarrollo de las labores se enmarca dentro de la estructuración global de los recursos y poblacio-

\footnotetext{
${ }^{24}$ El estudio de los lindones de la Fuente de la Mora se ha completado con los datos procedentes de los análisis de las muestras de suelos recogidas en los perfiles de los cortes. Estos datos completan los resultados antes expuestos, aunque su importancia y representatividad se verá aumentada con la ampliación del muestreo a otros suelos de la misma zona, lo que permitirá poseer un cuadro edafológico completo que actúe de «trama explicativa» base para el estudio de la organización y explotación de este territorio de acuerdo con nuestra escala de trabajo. La importancia de la realización de este tipo de análisis y su aplicación en estudios territoriales se ha puesto de relieve en algunos trabajos que muestran como la utilización de la cartografía de suelos y la realización de muestreos sistemáticos puede ser un útil rentable para el estudio de paisajes agrarios (Gaiffe, 1998).
}

nes de la zona que se reordenan en función de la explotación del yacimiento aurífero ${ }^{25}$. El inicio de los trabajos en la zona exigió una planificación integral del espacio que debió tener en cuenta todos recursos necesarios para su mantenimiento, como parece reflejarse en el aprovechamiento de la red hidraúlica destinada al laboreo minero para el abastecimiento de las labores agrícolas.

El trazado de la red hidráulica es lo primero que se construye antes de iniciar la explotación del yacimiento aurífero, pero lógicamente las estructuras que se han conservado corresponden, tanto si se consideran de forma global como si se analizan más detalladamente por sectores, a la última fase de explotación. Un buen conocimiento de la red hidráulica y de sus modificaciones a lo largo de toda la explotación son esenciales para definir el desarrollo de los trabajos mineros. Las labores no son obras planificadas de antemano en su totalidad, su desarrollo progresivo hizo necesario que los técnicos romanos fuesen construyendo nuevas infraestructuras hidráulicas a medida que se iban desplazando de un sector de laboreo a otro. De hecho, los diversos depósitos de explotación y sus correspondientes canales emisarios o de explotación nos sirven para marcar la separación entre los diferentes sectores.

La red hidráulica está formada por los canales y depósitos que aportan y regulan el agua necesaria para la explotación, ya que el agua es utilizada sucesivamente para deshacer el conglomerado aurífero, arrastrarlo, lavarlo y evacuar los estériles resultantes. En la reconstrucción propuesta de la red hidráulica de Las Cavenes se han distinguido los siguientes tipos de canalizaciones y zonas de embalsamiento de agua ${ }^{26}$ :

- Canales de abastecimiento, corrugi o canales ${ }^{27}$, que son los que generan una nueva red hidráulica; parten desde la propia captación de agua o desde los depósitos reguladores y desembocan en sectores de explotación, generalmente con sus correspondientes depósitos. Su trazado fue modificado

${ }_{25}$ No es mucho lo que sabemos de la distribución del poblamiento romano en esta zona. Los sondeos realizados por nosotros en La Corona, uno de los lugares que Morán vincula a Las Cavenes (ver nota 2), no han proporcionado ningún tipo de evidencia material que permita la confirmación de un asentamiento en este lugar.

${ }_{26}$ Todas las estructuras se han numerado en un orden teóricamente ascendente de modernidad, de forma que el depósito d-2 es potencialmente más antiguo que el d-12, el canal de abastecimiento c- 2 más antiguo que el c-4 y el sector S-2 más que el S-8. Todo ello dentro de la relatividad a la que nos hemos referido.

${ }^{27}$ Los términos latinos utilizados siguen la nomenclatura empleada por Plinio el Viejo en la ya mencionada descripción de las minas de oro de Asturia, Gallaecia y Lusitania (Plin., NH, 33, 66-78). 


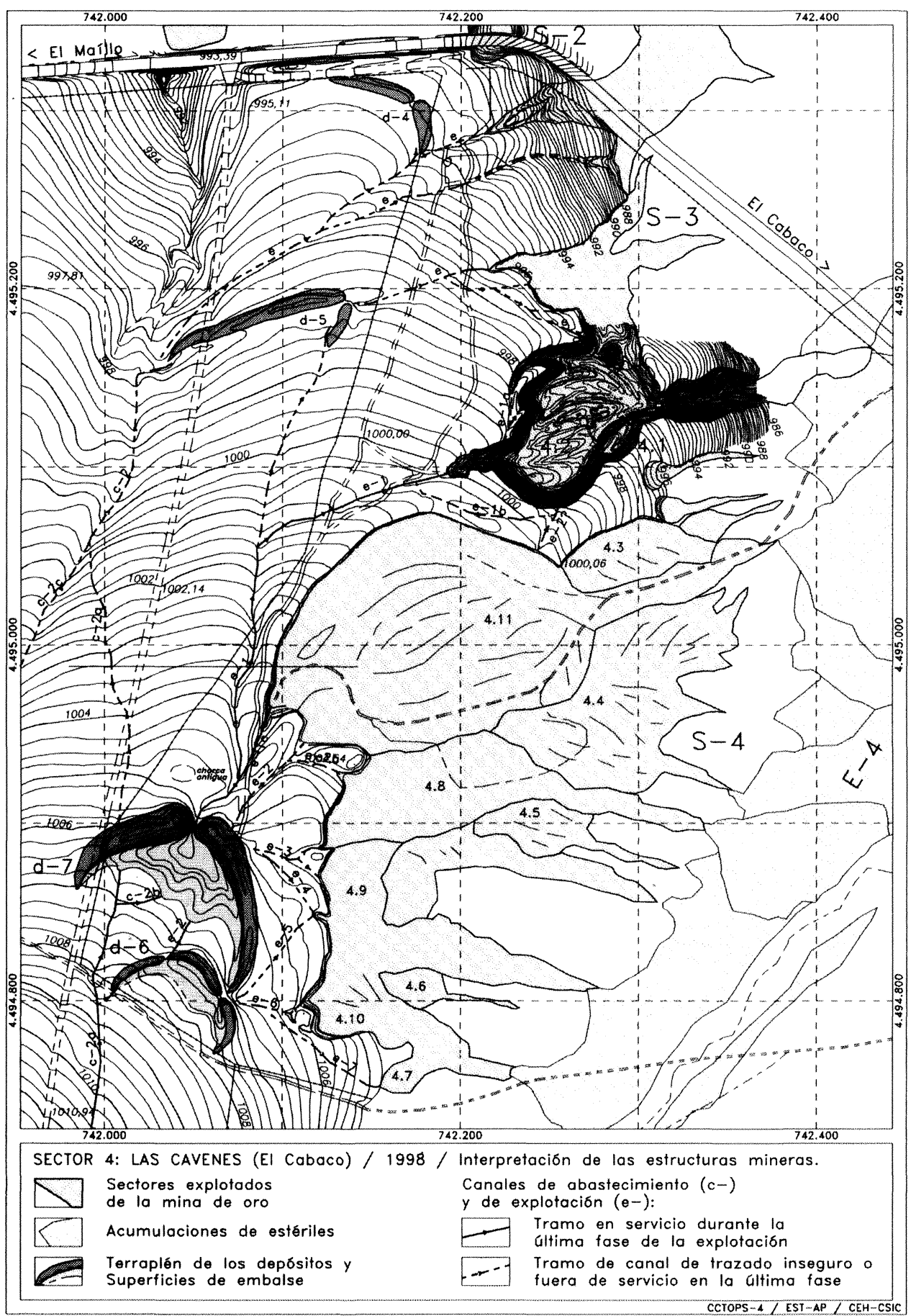

Fig. 8.-Plano topográfico del Sector 4 de Las Cavenes de El Cabaco (escala: 1/4.000, equidistancia entre curvas: $25 \mathrm{~cm}$ ).

a medida que se desarrollaba la explotación, de forma que es difícil, como se ha dicho, establecer el recorrido exacto de los correspondientes a las primeras fases.

La captación de agua se producía en los arroyos que nacen inmediatamente al sur, en las faldas de la Peña de Francia. Aunque se ha realizado ya un estudio de las evidencias visibles de esta red de abastecimiento (Sánchez-Palencia, 1997), sería demasiado prolijo describirla ahora en extensión. Como 
resumen, hay que resaltar la evidencia de dos canales de abastecimiento, al menos, que llegan hasta la zona más alta de las labores desde el suroeste y sureste respectivamente. También parece claro que la Reguera, una acequia de abastecimiento actual para El Cabaco, se superpone, y reutiliza al menos en parte un canal de abastecimiento romano (fig. 3).

- Los depósitos de agua, piscinae o stagna, actúan como reguladores de la corriente suministrada por los canales de abastecimiento y envían el agua a los diferentes frentes de laboreo. La apertura de un corte estratigráfico longitudinal en uno de los depósitos, el depósito de explotación d-7, ha permitido la individualización de los diferentes elementos que componen su estructura. La sencillez de la forma exterior del depósito contrasta con sus características constructivas, bastante complejas. Su construcción requirió una serie de trabajos previos de acondicionamiento del terreno. Una vez que se planificó y delimitó el área que debía ocupar el depósito, su superficie fue preparada con varios niveles de distinta potencia y composición sobre los que se asentó el terraplén que sirve de dique de contención. Los análisis mineralógicos de las arcillas procedentes de las distintas capas que conforman la estructura del depósito muestran que se realizó una selección de los materiales para lograr la máxima impermeabilidad del embalse.

Es bastante frecuente en Las Cavenes la disposición emparejada de los depósitos. Este es el caso del sector $\mathrm{S}-4$, en el que se han centrado nuestros trabajos de excavación y sondeo, y donde existen dos depósitos, el d-6 y d-7, abastecidos ambos a través del ramal c-2a del canal de abastecimiento c-2, que enlaza con la red hidráulica principal de captación usada para todo el yacimiento aurífero. La realización de una detallada topografía sobre el terreno (a escala $1 / 400$ y $1 / 800$ y con una equidistancia entre curvas de nivel de $25 \mathrm{~cm}$ ), completada con los datos procedentes de la fotointerpretación de las fotografías aéreas (figs. 9 y 10) de la zona ha permitido comprobar que la construcción del d-7 sobre el d-6 respondió a la necesidad de un mayor caudal de agua (fig. 12). Esta modificación en la red hidráulica nos indica el carácter empírico y pragmático que guiaba en gran medida la explotación romana, puesto que los ingenieros romanos se vieron obligados a construir un nuevo depósito para poder explotar una zona cuya extensión y riqueza seguramente no controlaban bien desde el principio.

- Los canales emisarios o de explotación, emissaria (denominados con la letra «e» en las figs. 8-11), son los que, con una longitud mayor o menor y con diversas ramificaciones a veces, salen de los depósitos hacia los frentes de explotación de las distintas labores donde desembocan.

La configuración de Las Cavenes no deja lugar a dudas sobre el sistema de explotación empleado. Los desmontes o vaciados mineros producidos sobre el yacimiento conforman la mina de oro propiamente dicha, la zona concreta de donde se extrajo el oro. Están delimitados en su cabecera por los frentes de explotación o tajos de laboreo que marcan la extensión máxima de la mina. En la parte opuesta o terminal se hallarían los canales de lavado o agogae que marcarían el fin del desmonte. Aunque su construcción en madera ha impedido lógicamente la conservación de estos últimos, sí se puede identificar el final de las zonas de extracción gracias al estrechamiento que comúnmente marca su situación aproximada y el inicio del canal de evacuación de estériles. La superficie interna de todas las labores muestra hileras de cantos rodados que testimonian la separación de los estériles más gruesos antes de la posición que ocuparía el canal de lavado. A una escala más amplia, se puede comprobar la convergencia de todos los surcos hacia un mismo punto y la formación a una cierta distancia de él de las colas de lavado o conos de deyección donde se acumulaban los estériles más finos (fig. 8).

De acuerdo con las anteriores evidencias, el sistema de explotación empleado en Las Cavenes fue el selectivo mediante «series de surcos convergentes». En resumen, consiste en ir arrojando agua desde los canales emisarios o de explotación sobre surcos que se trazan en el suelo y que van erosionando sucesivamente el conglomerado aurífero y acarreándolo en forma de lodos hacia los canales de lavado o agogae donde quedaría depositado el oro. El sistema de explotación es bastante homogéneo, puesto que las características topográficas y geológicas del yacimiento aurífero son también bastante uniformes ${ }^{28}$. La topografía con que se encontraban los ingenieros romanos era también muy similar en todos los casos. Se trataba de aprovechar el desnivel del borde amesetado y utilizar esa pendiente para potenciar el valor erosivo de la fuerza hidráulica.

\footnotetext{
${ }^{28}$ El yacimiento aurífero beneficiado en Las Cavenes está formado por los depósitos conglomeráticos que constituyen las «series rojas neógenas» de la zona. Geomorfológicamente, se trata de la raña que conforma el techo sedimentario en el piedemonte de la Sierra de Francia. Son conglomerados depositados en régimen de abanicos aluviales, con cantos rodados de cuarcita fundamentalmente y con una matriz más arenosa en los niveles superficiales y más arcillosa en los inferiores. La zona explotada en época romana presenta unos cantos rodados subangulares o angulares, es decir, que no han sufrido mucho transporte, ya que se halla en la zona proximal de los abanicos, muy cerca de su área madre.
} 

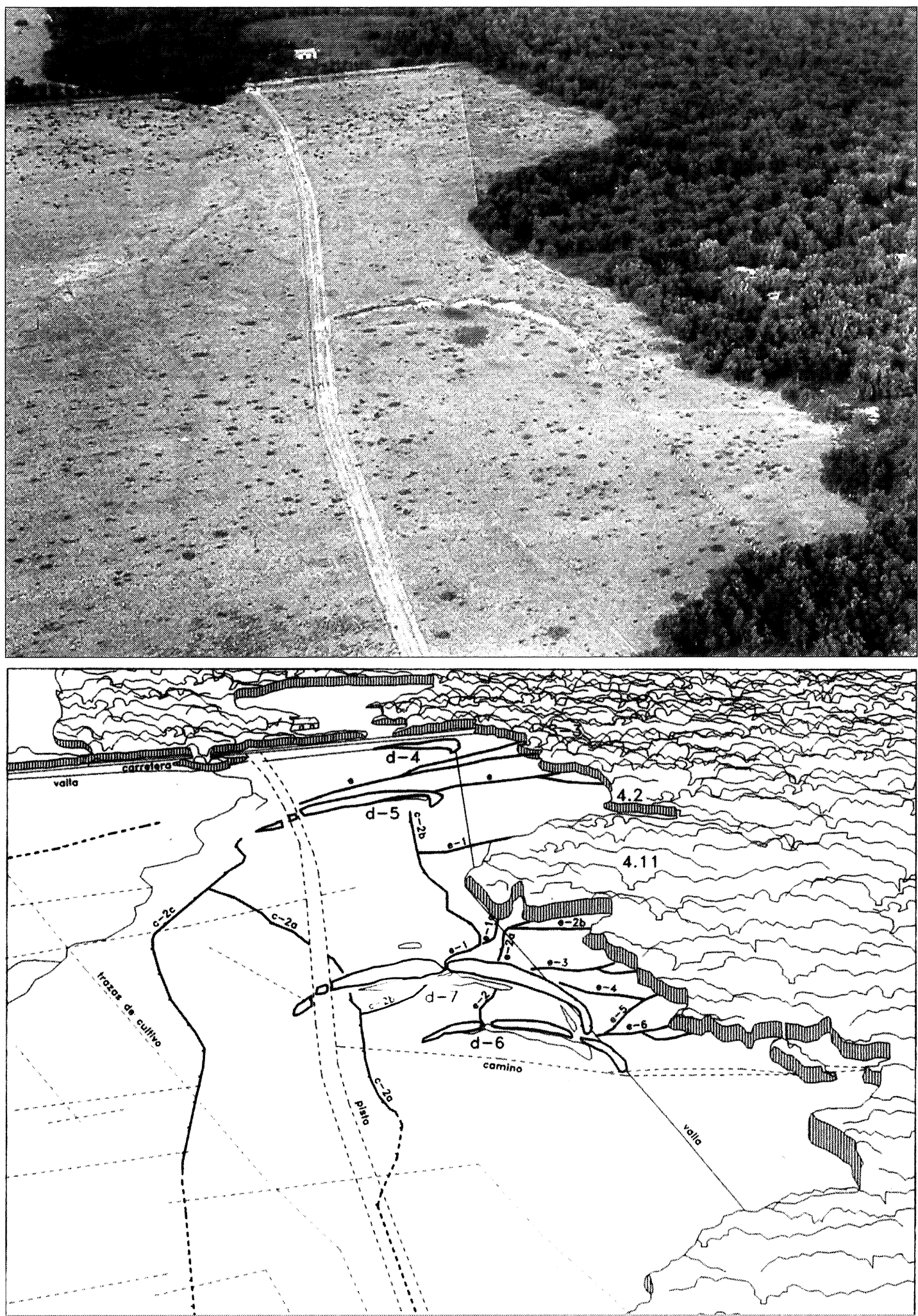

Fig. 9.-Fotointerpretación desde el sur de la red hidráulica inmediata al Sector 4 de Las Cavenes de El Cabaco. 

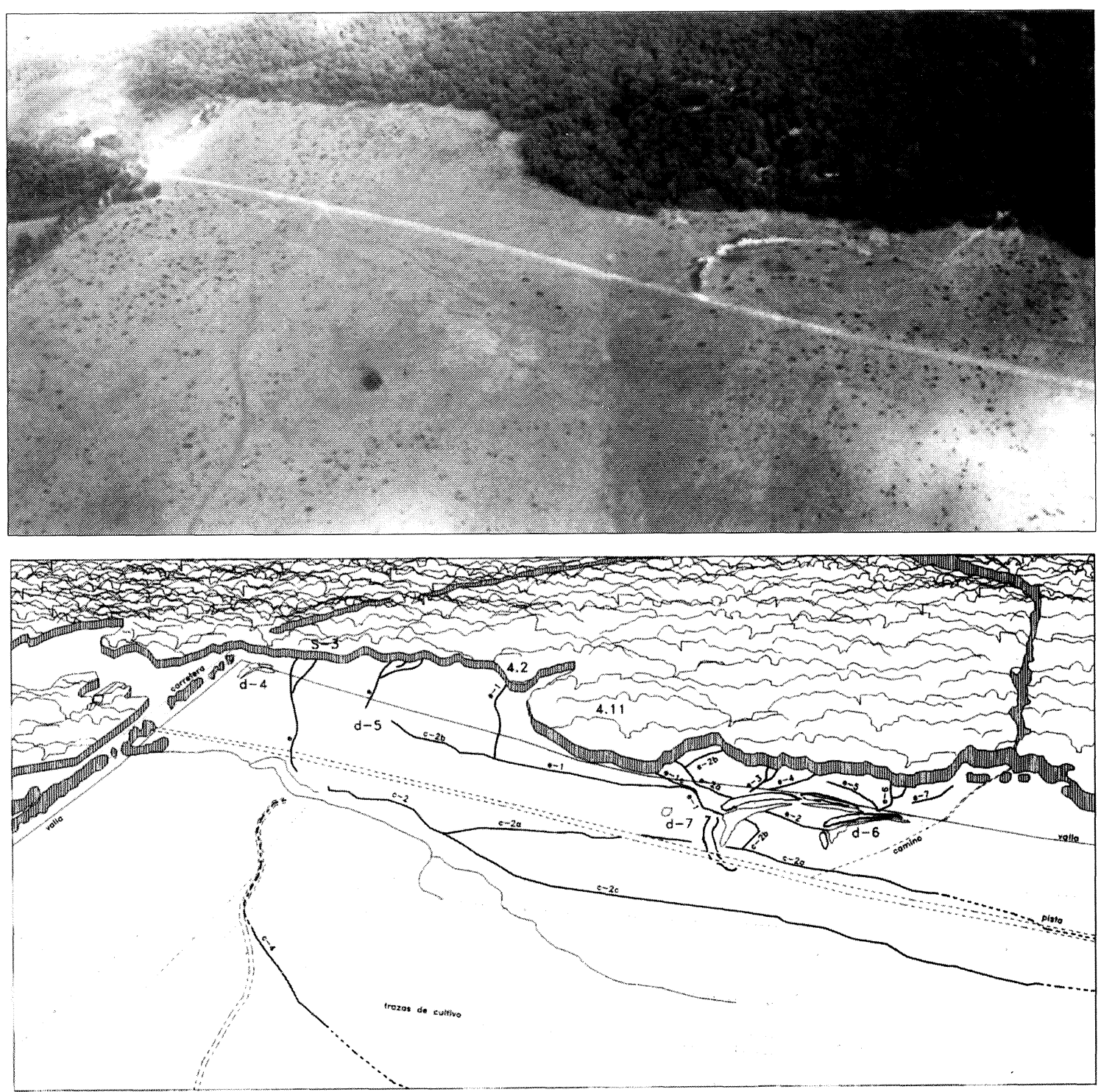

Fig. 10.-Fotointerpretación desde el oeste de la red hidráulica inmediata al Sector 4 de Las Cavenes de El Cabaco.

Al ser relativamente pequeña la potencia media de la raña explotada, unos $5 \mathrm{~m}$ aproximadamente, es coherente que el sistema de explotación elegido fuera selectivo (Sánchez-Palencia y Orejas, 1994, 159 ss). Este tipo de sistema se contrapone a los que denominamos extensivos y, frente a ellos, se caracteriza por ir removiendo y lavando exhaustivamente y de forma sistemática toda la potencia del conglomerado poco a poco, en sucesivas acciones de la fuerza hidráulica como agente erosivo y extractor del conglomerado ${ }^{29}$. Lógicamente, las técnicas de

${ }^{29}$ Preferimos utilizar la diferenciación general entre técnicas de explotación selectivas y extensivas en vez de otras de explotación selectiva indican una relativa riqueza aurífera del terreno beneficiado.

El avance de la mina exigía poder evacuar permanentemente la gran mayoría del material estéril fuera del yacimiento, de forma que el posible frente de explotación quedase siempre limpio y libre para la extracción. Esto se conseguía mediante la excavación de unos canales de evacuación por donde se hacía salir casi todo el material removido, una vez lavado y recogido todo el oro que contenía. Como se ha señalado antes, el material más grueso y pe-

carácter más formalista. Para la comparación entre las nomenclaturas propuestas sobre las diversas técnicas: SánchezPalencia y Orejas, 1994, 159-162. 


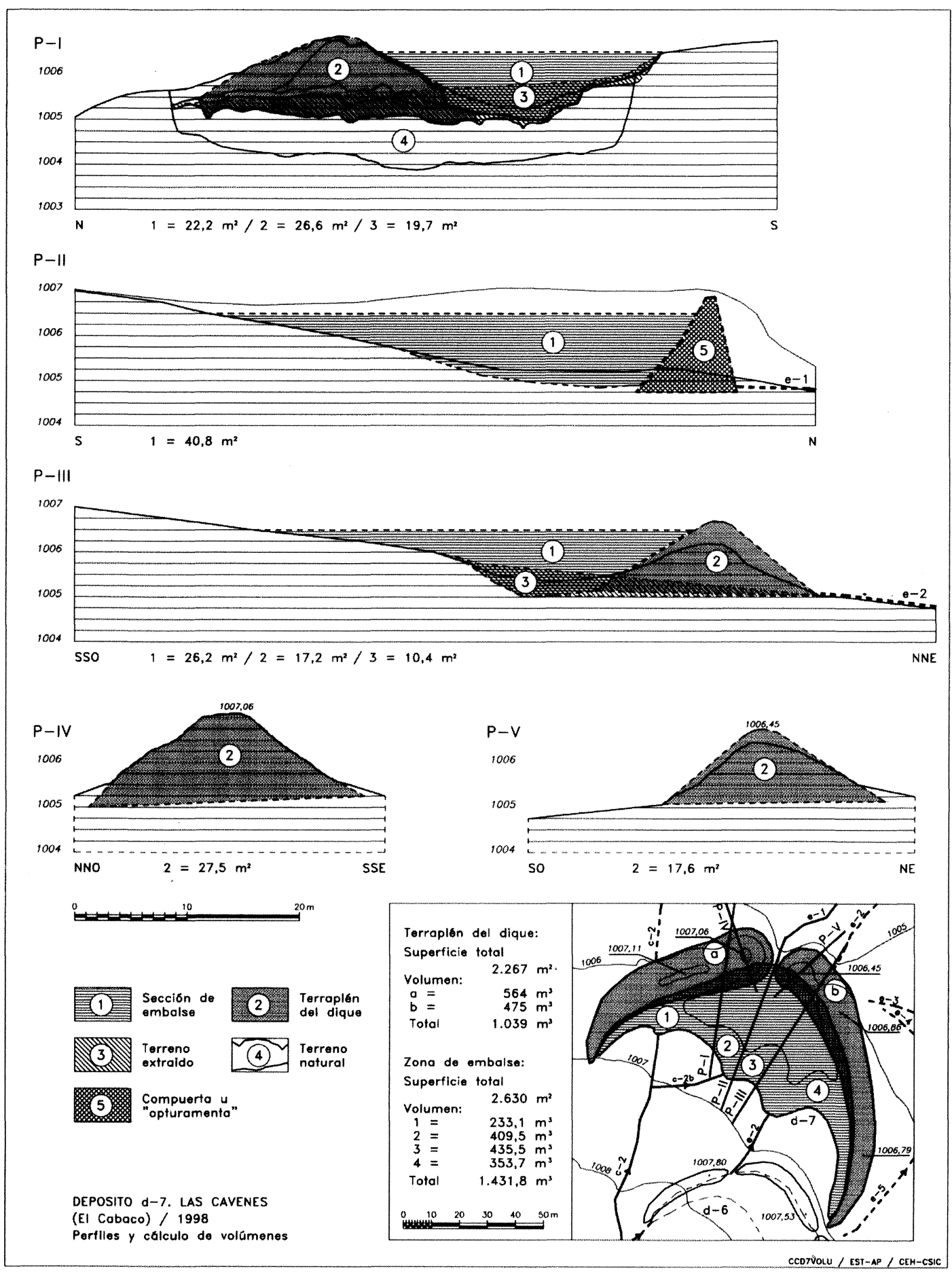

Fig. 11.-Perfiles y cálculo de volúmenes del depósito d-7 de Las Cavenes de El Cabaco.

sado era el único que quedaba depositado en el interior de la labor minera. Como se puede apreciar en las figuras 3 y 8 prácticamente cada labor dio lugar a una acumulación de estériles. El mayor o menor encajonamiento de las vaguadas donde se acumularon hace que los conos de deyección sean más o menos difusos o nítidos.

La carencia de elementos de datación absolutos 
dificulta la reconstrucción del proceso concreto que siguió la explotación romana en Las Cavenes. El descubrimiento del yacimiento aurífero debió de producirse a través de los placeres fluviales existentes dentro de la propia cuenca. Las trincheras de prospección que se documentan en la zona más septentrional del yacimiento (T-1 a T-5) o incluso más al sur (T-6) (figs. 3 y 8), entre la zona más fuertemente explotada, permiten deducir un intenso trabajo de prospección romana antes del inicio de los trabajos sistemáticos de explotación. Este tipo de sondeo, que lógicamente sólo se habría conservado en aquellas zonas que luego no fueron objeto de explotación intensa, permitiría de sobra ir delimitando las áreas donde la ley de oro era más interesante.

Las actuaciones llevadas a cabo en 1998 han proporcionado abundante y novedosa información sobre el desarrollo del laboreo minero, tanto de carácter cualitativo como cuantitativo. Profundizar mínimamente en esta información requeriría un espacio excesivo para los objetivos de este primer trabajo sobre Las Cavenes, por lo que consideramos más apropiado hacerlo en otro momento. Vamos a conformarnos simplemente con resaltar algunos de los resultados más destacados.

Los estudios detallados sobre el Sector 4 de las labores han confirmado la cronología relativa propuesta para toda la mina de oro, reflejada en la numeración de las diferentes estructuras según ya se ha indicado. La microtopografía del levantamiento realizado, que afecta en particular a los depósitos d6 y d-7 y a las labores 4.1 y 4.2 , ha facilitado datos muy precisos sobre la relación entre el volumen de agua y el material removido y sobre la ejecución de las técnicas de explotación selectivas mediante surcos convergentes, así como sobre la pendiente y disposición de dichos surcos y el canal de lavado o agoga. A partir de estos últimos estudios se han podido matizar también las primeras valoraciones realizadas sobre el volumen de material removido en toda la mina (Sánchez-Palencia, 1997), que podría estar en torno a los 1'53 $\mathrm{Mm}^{3}$. Para remover toda esta cantidad de material se construyó una red hidráulica que, según lo hasta ahora documentado, no es inferior a los $20,5 \mathrm{~km}$.

\section{Consideraciones finAles}

Uno de los aspectos más interesantes de la investigación llevada a cabo en Las Cavenes de El Cabaco es la documentación conjunta de estructuras mineras y acondicionamientos agrarios en una misma zona. Esta evidencia viene a ratificar que la activi- dad minera romana nunca tuvo un carácter sectorial y que su puesta en práctica ha de entenderse dentro de una concepción global de la explotación del territorio. Las fechas proporcionadas por la cerámica indican que la organización sistemática de tal explotación no se produjo hasta bien entrado el siglo I d.C., aunque el contexto histórico a una escala comarcal y en particular los testimonios epigráficos sobre la delimitación entre las ciuitates más próximas hacen remontar las primeras medidas de reorganización hasta la época de Augusto. Estas circunstancias son prácticamente las mismas que se han documentado en las zonas mineras, de mayor envergadura y extensión, del noroeste peninsular. Hay que considerar por lo tanto que la vinculación que estableció Plinio el Viejo entre las minas de oro astures, galaicas y lusitanas se produjo ya desde los inicios del laboreo y respondió a una misma política. La complejidad de los trabajos necesarios para beneficiar el oro justificaba plenamente un período relativamente amplio de preparación. En Las Cavenes se puede apreciar claramente el alcance de este primer momento a través de las diversas trincheras de prospección y, sobre todo, por los trabajos de planificación, captación y trazado de la imprescindible red hidráulica. En relación con esta última, los trabajos en Las Cavenes han demostrado también el carácter empírico de la tecnología romana, que condujo a continuas modificaciones de los canales y depósitos en función de la ley del yacimiento.

En este primer avance de resultados queda patente que la investigación en Las Cavenes no ha hecho más que empezar. Uno de los objetivos prioritarios ha de ser el estudio del poblamiento y por lo tanto la realización de prospecciones sistemáticas en una área suficientemente amplia. Hay que recordar que las labores mineras y los acondicionamientos agrarios del tipo de los «lindones» cuentan ya con varios indicios claros en las inmediaciones. La prospección ha completarse por otro lado con la excavación de algún núcleo. Parece evidente por el material cerámico recogido en los sondeos que en las inmediaciones de La Fuente de la Mora, seguramente inmediatamente al oeste, ha de existir un asentamiento, cuyo estudio puede proporcionar importantes datos sobre la población involucrada en la explotación de los recursos mineros y agrícolas. Este último aspecto es de capital importancia si se quieren precisar los mecanismos de articulación que estableció el Estado romano entre sus intereses mineros y la reorganización de las ciuitates del territorio vetón.

En fin, no queremos olvidar el alcance patrimonial de las actuaciones realizadas y a realizar. En 
este momento ya se cuenta con un itinerario señalizado que informa al visitante de los aspetos más relevantes de la minería romana en su Sector 4 . Nuestro equipo está trabajando en colaboración con el Ayuntamiento de El Cabaco, cuyo interés por los trabajos arqueológicos desarrollados es ciertamente ejemplar, y con la empresa Desarrollo de Recursos Geológicos para completar y ampliar esta labor de proyección social de la zona minera de Las Cavenes y de otros valores culturales o naturales del entorno. En último término se trata de investigar y explotar racionalmente un auténtico Paisaje Cultural, de modo que contribuya al desarrollo de una zona con un indudable potencial turístico.

\section{BIBLIOGRAFÍA}

Álvarez Sanchís, J. R., 1999: Los Vettones. Real Academia de la Historia. Madrid.

Ariño Gil, E. y García de Figuerola Paniagua, M., 1993: Un terminus de agrimensor de carácter técnico procedente de la Sierra de Gata (Villamiel, Cáceres). AEspA, 66, 1993, 258-265.

Ariño Gil, E. y Rodríguez Hernández, J., 1997: El poblamiento romano y visigodo en el territorio de Salamanca. Datos de una prospección intensiva. Zephyrus, 50, 1997, 225-245.

Bonneau, M. y Souchier, B. (eds.), 1987: Edafología. 2. Constituyentes y propiedades del suelo. Barcelona.

ButZER, K.W., 1976: Geomorphology from the Earth. Nueva York.

Butzer, K.W., 1989: Arqueología, una ecología del hombre: método y teoría para un enfoque contextual. Barcelona ( $1^{\text {a }}$ edición en inglés: 1982).

Chapa Brunet, T. y Mayoral Herrera, V., 1998: Explotación económica y fronteras políticas: diferencias entre el modelo ibérico y el romano en el límite entre la alta Andalucía y el sureste, AEspA, 71, 63-77.

Chouguer, G. y Favory, F., 1991: Les paysages de l'Antiquité. Terres et cadastres de l'occident romain (IVe s. avant J.-C./III s. après J.C.). Paris.

Courty, M.A.; Goldberg, P. y MacPhall, R., 1989: Soils and micromorphology in archaeology. Cambridge U.P.

Domergue, C., 1987: Catalogue des mines et fonderies antiques de la Péninsule Ibérique (I y II). Madrid.

Domergue, C., 1990: Les mines de la Péninsule Ibérique dans l'Antiquité romaine. Roma.

EDMONSON, J.C., 1990a: Romanization and urban development in Lusitania. The early Roman em- pire in the West. T. Blagg y M. Millet (eds.), Oxford, 151-178.

Edmonson, J.C., 1990b: Le garum en Lusitania urbaine et rurale: hiérarchies de demande et de production. Les villes de Lusitanie Romaine. Hiérarchies et territoires. Table ronde internationale du CNRS (Talence, 8-9 décembre, 1988). J.G. Gorges (ed.), Paris. 124-141.

EDMONSON, 1992-93: Creating a provincial landscape: Roman imperialism and rural change in Lusitania, Actas de la mesa redonda internacional El medio rural en Lusitania romana, formas de habitat y ocupación del suelo (J.G. Gorges; M. Salinas de Frías, eds.) = Studia Historica Historia Antigua, X-XI, 13-30.

Francisco Martín, J. de, 1989: Conquista y romanización de Lusitania. Acta Salmanticensia. Ests. históricos y geográficos, 58. Univ. de Salamanca. Salamanca.

GaIffe, M., 1998: La cartographie pédologique au service de l'étude des cadastres. Atlas historique des Cadastres d'Europe. M. Clavel-Lévêque y A.Vignot (dirs.). Bruselas.

Gandullo, J.M., 1984: Clasificación básica de los suelos españoles. Madrid.

Gómez-Moreno, M., 1967: Catálogo Monumental de España. Provincia de Salamanca. Madrid.

GonZÁlez-FernándeZ, Ma.L., 1999: De campamento a ciuitas. La primera fortificación urbana de Asturica Augusta (Astorga, León). Numantia, 7, 95-115.

Iglesias, L.; Rodríguez, Mª B. y Marcos, M.S., 1991: Arqueología y Prehistoria de Salamanca: Intervenciones y bibliografía actualizada. Del Paleolítico a la Historia (Museo de Salamanca). Salamanca, 175-201.

LE Roux, P., 1982: L'armée romaine et l'organisation des provinces ibériques d'Auguste à l'invasion de 409. Paris.

Le Roux, P., 1990: Les villes de statut municipal en Lusitanie romaine. Les villes de Lusitanie romaine. Hiérarchies et territoires. Table ronde internationale du CNRS (Talence, 8-9 décembre, 1988). J.G. Gorges (ed.), Paris. 35-47.

LE Roux, P., 1994: Cités et territoires en Hispanie: l'épigraphie des limites, Mélanges de la Casa de Velázquez, XXX (1), 37-51.

MGE, h. $\mathrm{n}^{\circ}$ 527, Tamames, 1990: Mapa geológico de España. escala 1:50.000. Hoja $n^{\circ}$ 527. Tamames. Inst ${ }^{\circ}$ Tecnológico GeoMinero de España. Madrid.

Maluquer de Motes, J., 1956: Carta Arqueológica de España. Salamanca. Salamanca.

MANGAS, J. 1992: Ciudades antiguas de la provincia 
de Salamanca (siglo III a.C.-Diocleciano). Actas I Congreso de Historia de Salamanca, I. Salamanca.

Mangas, J. y Solana, J.Ma., 1985: Historia de Castilla y León, 2. Romanización y germanización de la Meseta Norte. Valladolid.

MoRÁn, C., 1940: Mapa histórico de la provincia de Salamanca. Salamanca.

Morán, C., 1946: Reseña histórico-artística de la provincia de Salamanca. Acta Salmanticensia. Filosofía y Letras, II, 1. Univ. de Salamanca. Salamanca.

OREJAs, A., 1996: Estructura social y territorio. El impacto romano en la cuenca noroccidental del Duero, Anejos de Archivo Español de Arqueología, 15. Madrid.

Orejas, A. y Sánchez-Palencia, F.-J., en prensa: La arqueología de la conquista del noroeste de la Península Ibérica. Actas del II Congreso de Arqueología Peninsular, Zamora.

Orejas, A.; Sastre, I., 1999: Fiscalité et organisation du territoire dans le nord-ouest de la Péninsule Ibérique: ciuitates, tribut et ager mensura comprehensus. Dialogues d'Histoire Ancienne, 25.2.

Porta, J.; López-Acevedo, M. y Roquedo, C., 1994: Edafología. Para la agricultura y el medio ambiente. Madrid.

Roldán, J.M., 1968: Fuentes antiguas para el estudio de los vettones. Zephyrus, XIX-XX, 73-106.

Roldán, J.M., 1971: Iter ab Emerita Asturicam. El Camino de la Plata. Salamanca.

RoldÁn, J.M., 1974: Hispania y el ejército romano. Contribución a la historia social de la España Antigua. Salamanca.

Recursos Minerales, 1988: Los Recursos Minerales de Castilla y León. $N^{o}$ 1. Oro. Valladolid.

SALINAS DE FRÍAS, M., 1986: La organización tribal de los vettones. Salamanca.

Salinas de Frías, M., 1989: Sobre las formas de propiedad comunal de la cuenca del Duero en época prerromana, Veleia, 6, 103-110.

Salinas de Frías, M., 1990: Las ciudades romanas de Lusitania oriental: su papel en la transformación del territorio y la sociedad indígena. Les villes de Lusitanie romaine. Hiérarchies et territoires. Table ronde internationale du CNRS ( Talence, 8-9 décembre, 1988). J.G. Gorges, ed., Paris. 255-264.

Salinas de Frías, M., 1992: El elemento romano de Salamanca durante el Alto Imperio. Actas I congreso de Historia de Salamanca, I, Salamanca, 301-309.
SALINAS DE FríAS, M., 1992-93: El poblamiento rural antiguo de la provincia de Salamanca: modelos e implicaciones históricas. Actas de la mesa redonda internacional «El medio rural en Lusitania romana, formas de hábitat y ocupación del suelo». J.G. Gorges; M. Salinas de Frías, eds.=Studia Historica. Historia Antigua, X-XI, 177-188.

SÁnCHEZ-PAlEncia, F.-J., 1983: La explotación del oro de Asturia y Gallaecia en la Antigüedad. Universidad Complutense de Madrid (Tesis doctoral inédita).

Sánchez-Palencia, F.-J., 1989: La explotación del oro en la Hispania romana: sus inicios y precedentes, Minería y Metalurgia en las antiguas civilizaciones mediterráneas y europeas. Coloquio internacional asociado, Madrid, II, 35-52.

Sánchez-Palencia, F.-J., 1997: Minería de oro romana en Lusitania: Las Cavenes de El Cabaco (Salamanca), Madrid (estudio inédito).

Sánchez-Palencia, F.-J. y Orejas, A., 1994: La Minería de oro del noroeste peninsular. Tecnología, organización y poblamiento, Minería y Metalurgia en la España prerromana y romana (D. Vaquerizo Gil, coord.). Actas de los Seminarios de verano «Fons Mellaria 1992" (Fuenteovejuna, Córdoba), 147-223.

Sánchez-Palencia, F.-J. y Pérez, L.C., 1989: Los yacimientos auríferos de la Península Ibérica. Posibilidades de explotación en la Antigüedad, El oro en la España Prerromana. Monografía de la Rev. de Arqueología. Madrid, 16-22.

Sánchez-Palencia, F.-J.; Fernández-Posse, M.D.; Fernández Manzano, J. y Orejas, A., 1996: La zona arqueológica de Las Médulas (León). Guía arqueológica. Valladolid.

Santonja, M., 1991: Comentarios generales sobre la dinámica del poblamiento antiguo en la provincia de Salamanca. Del Paleolítico a la Historia. Salamanca, 14-31.

Sastre, I., 1998: Formas de dependencia social en el noroeste peninsular (Transición del mundo prerromano al romano y época altoimperial). Ponferrada.

SAYAS, J.J., 1979: Algunas consideraciones sobre el origen de Lusitania como provincia. Estudios dedicados a Carlos Callejo Serrano. Cáceres, 737752.

TIR K-29, 1991: Tabula Imperii Romani. Hoja K29: Porto. Conimbriga - Bracara - Lucus - Asturica. Balil, A.; Pereira, G.; Sánchez-Palencia, F.J. (eds.). Madrid.

Vigil, M., 1973: Historia de España Alfaguara. Edad Antigua. Madrid. 